Please share your stories about how Open Access to this article benefits you.

\title{
Thermochronologic constraints on the late Cenozoic exhumation history of the Gurla Mandhata metamorphic core complex, Southwestern Tibet
}

by A. T. McCallister et al.

2013

This is the published version of the article, made available with the permission of the publisher. The original published version can be found at the link below.

A. T. McCallister et al. (2013). Thermochronologic constraints on the late Cenozoic exhumation history of the Gurla Mandhata metamorphic core complex, Southwestern Tibet. Tectonics

Published version: http://www.dx.doi.org/10.1002/2013TC003302

Terms of Use: http://www2.ku.edu/ scholar/docs/license.shtml 


\section{Tectonics}

\section{RESEARCH ARTICLE}

10.1002/2013TC003302

Key Points:

- Fast Exhumation (4-5 mm/yr) since 12 Ma

- Similar timing, rate and fault slip as the Karakoram Fault

- 8 Ma Zr (U-Th)/He

Thermochronometic ages across the range

Correspondence to:

M. H. Taylor,

mht@ku.edu

\section{Citation:}

McCallister, A. T., M. H. Taylor, M. A. Murphy, R. H. Styron, and D. F. Stockli (2014), Thermochronologic constraints on the late Cenozoic exhumation history of the Gurla Mandhata metamorphic core complex, Southwestern Tibet, Tectonics, 33, 27-52, doi:10.1002/ 2013 TC003302.

Received 20 JAN 2013 Accepted 19 NOV 2013 Accepted article online 13 DEC 2013 Published online 12 FEB 2014

\section{Thermochronologic constraints on the late Cenozoic exhumation history of the Gurla Mandhata metamorphic core complex, Southwestern Tibet}

\author{
A. T. McCallister ${ }^{1}$, M. H. Taylor ${ }^{1}$, M. A. Murphy ${ }^{2}$, R. H. Styron ${ }^{1}$, and D. F. Stockli ${ }^{1,3}$ \\ ${ }^{1}$ Department of Geology, University of Kansas, Lawrence, Kansas, USA, ${ }^{2}$ Department of Earth and Atmospheric Sciences, \\ University of Houston, Houston, Texas, USA, ${ }^{3}$ Now at Jackson School of Geosciences, University of Texas-Austin, Austin, \\ Texas, USA
}

Abstract How the Tibetan plateau is geodynamically linked to the Himalayas is a topic receiving considerable attention. The Karakoram fault plays key roles in describing the structural relationship between southern Tibet and the Himalayas. In particular, considerable debate exists at the southeastern end of the Karakoram fault, where its role is interpreted in two different ways. One interpretation states that slip along the dextral Karakoram fault extends eastward along the Indus-Yalu suture zone, bypassing the Himalayas. The other interprets that fault slip is fed southward into the Himalayan thrust belt along the Gurla Mandhata detachment (GMD). To evaluate these competing models, the late Miocene history of the GMD was reconstructed from thermokinematic modeling of zircon (U-Th)/He data. Three east-west transects reveal rapid cooling of the GMD footwall from $8.0 \pm 1.3 \mathrm{Ma}$ to $2.6 \pm 0.7 \mathrm{Ma}$. Model simulations show a southward decrease in slip magnitude and rate along the GMD. In the north, initiation of the GMD range between 14 and $11 \mathrm{Ma}$ with a mean fault slip rate of $5.0 \pm 0.9 \mathrm{~mm} / \mathrm{yr}$. The central transect shows an initiation age from 14 to 11 Ma with a mean fault slip rate of $3.3 \pm 0.6 \mathrm{~mm} / \mathrm{yr}$. In the south, initiation began between 15 and 8 Ma with a mean fault slip rate of $3.2 \pm 1.6 \mathrm{~mm} / \mathrm{yr}$. The initiation ages and slip rates match the Karakoram fault across several timescales, supporting the idea that the two are kinematically linked. Specifically, the data are consistent with the GMD acting as an extensional stepover, with slip transferred southward into the Himalayas of western Nepal.

\section{Introduction}

The Tibetan plateau is the largest continental plateau on Earth with the final episode of collision between the Indian and Eurasian plates, and has been ongoing since the Early Cenozoic [e.g., Besse et al., 1984; Gaetani and Garzanti, 1991; Zhu et al., 2005]. Since the Middle Miocene, southern Tibet has been undergoing $\sim \mathrm{E}-\mathrm{W}$ extension while the Himalayan thrust belt has continuously accommodated arc-normal shortening, and a component of arc parallel shear in the High Himalaya. In the Himalaya and southern Tibet, structures accommodating arc-parallel extension have exhumed midcrustal rocks (Figure 1), such as the Ama Drime detachment-Nyonno detachment [Jessup et al., 2008; Langille et al., 2010; Jessup and Cottle, 2010], the Leo Pargil detachment [Thiede et al., 2006; Langille et al., 2012], the Karakoram fault [Searle et al., 1998; Zhang et al., 2011], and the Gurla Mandhata detachment (GMD) [Murphy et al., 2002]. These faults have accommodated $10 \mathrm{~s}$ of $\mathrm{km}$ of extension, are active, and are interpreted to interact with structures in the Himalayan thrust belt, and therefore are important in understanding strain patterns and the kinematic development of collision-related thrust belts.

\subsection{Models for Deformation of the Himalayan-Tibetan Orogen}

Several models have been proposed to explain how extension and strike-slip faulting within the Tibetan plateau are linked to the development of the Himalayan thrust belt. Lateral extrusion describes the eastward translation of Tibetan crust in response to the convergence between the Indian subcontinent and the southern margin of Asia. The model predicts a laterally continuous right-slip fault system along the Indus-Yalu suture (IYS) [Tapponnier et al., 1982; Peltzer and Tapponnier, 1988; Lacassin et al., 2004], which separates deformation within the Tibetan crust from that within the Himalayan thrust belt (Figure $2 \mathrm{~d}$ ). 


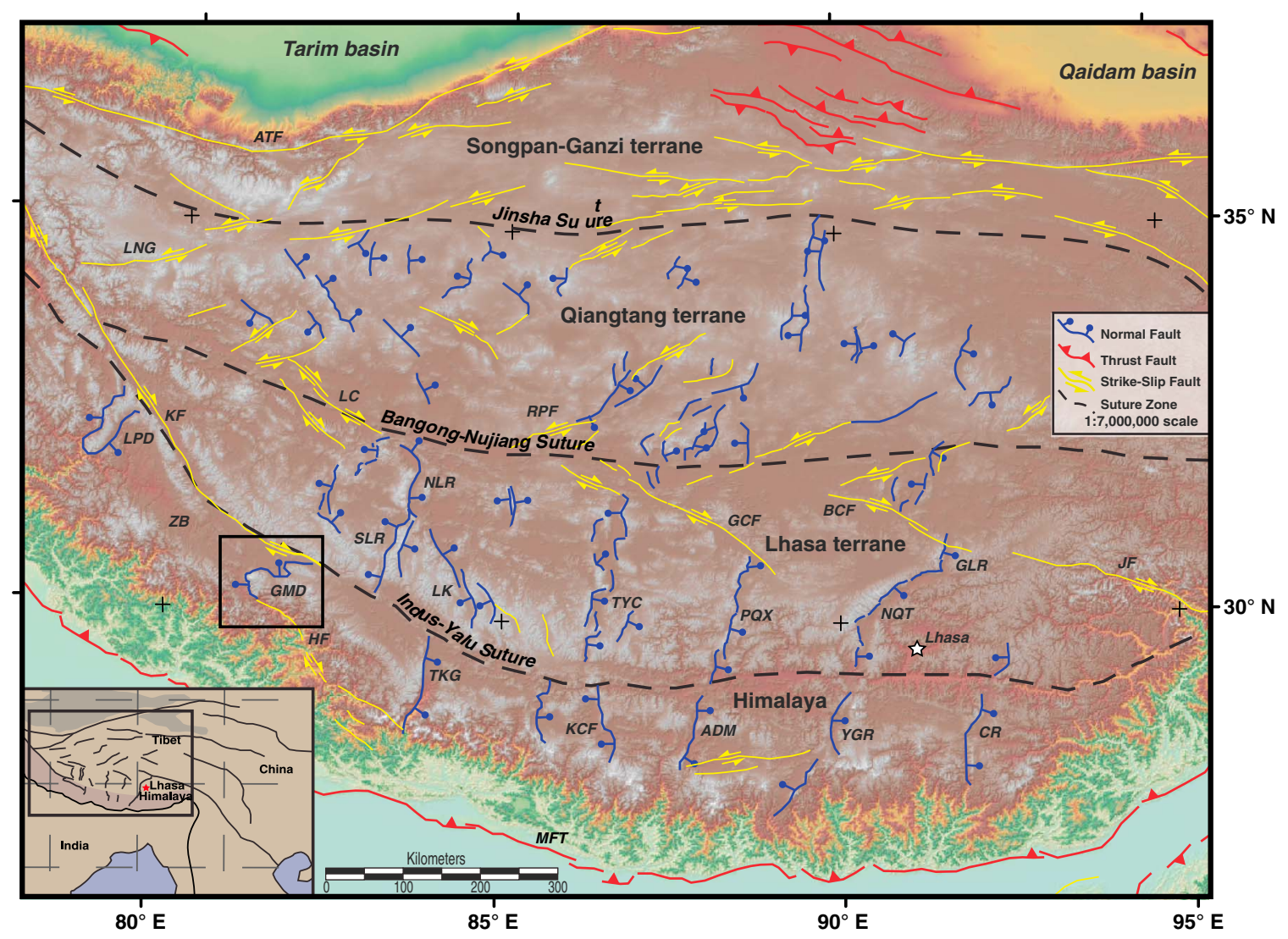

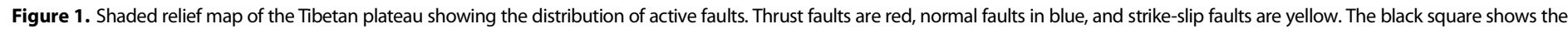

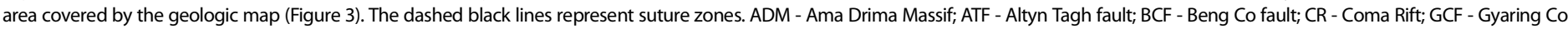

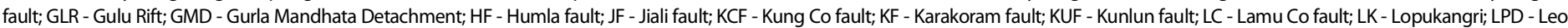

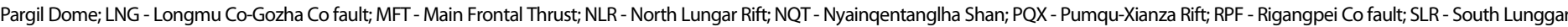

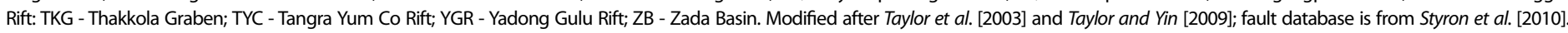

Oroclinal bending of the Himalayan thrust belt has been called upon to explain arc-parallel extensional patterns within the Himalaya and Tibet [Klootwijk et al., 1985; Ratschbacher et al., 1994], left-lateral strike-slip faulting in the eastern Himalaya (Figure 2a) [Ratschbacher et al., 1994; Li and Yin, 2008], and right-slip faulting in the western Himalaya. In the context of this model, the magnitude of extension is predicted to increase toward the central thrust front.

Radial spreading of the Himalaya arc has been called upon to explain the sequence of thrusting in the Himalayan thrust belt and maintenance of its arcuate geometry [Seeber and Armbruster, 1984; Molnar and LyonCaen, 1988; Murphy and Copeland, 2005]. The model predicts an increase in the circumference of the Himalayan arc, and an increase in the magnitude of extensional strain toward the hinterland of the Himalayan thrust belt (Figure $2 \mathrm{~b}$ ). Extension in the hinterland regions could be accommodated by a combination of extensional and strike-slip fault systems.

The oblique convergence model suggests that oblique convergence between India and the Himalayan arc is partitioned into an arc-normal component (thrust faulting) and an arc-parallel component (extensional and strike-slip faulting) [Seeber and Armbruster, 1984; Seeber and Pecher, 1998; McCaffrey and Nabelek, 1998]. The model predicts that right slip faulting dominates in the western portions of the Himalayan arc where convergence obliquity is highest, and north-south shortening is dominant in the central portions of the Himalayan arc where convergence is orthogonal (Figure 2c).

The models described above make specific predictions about the structural relationships occurring in southwestern Tibet, where fault systems within the Tibetan plateau are interpreted to interact with the Himalayan thrust belt. Considerable debate exists over which family of models best explains the pattern of active faulting in this region. For example, lateral extrusion states that slip along the Karakoram fault (KF) 


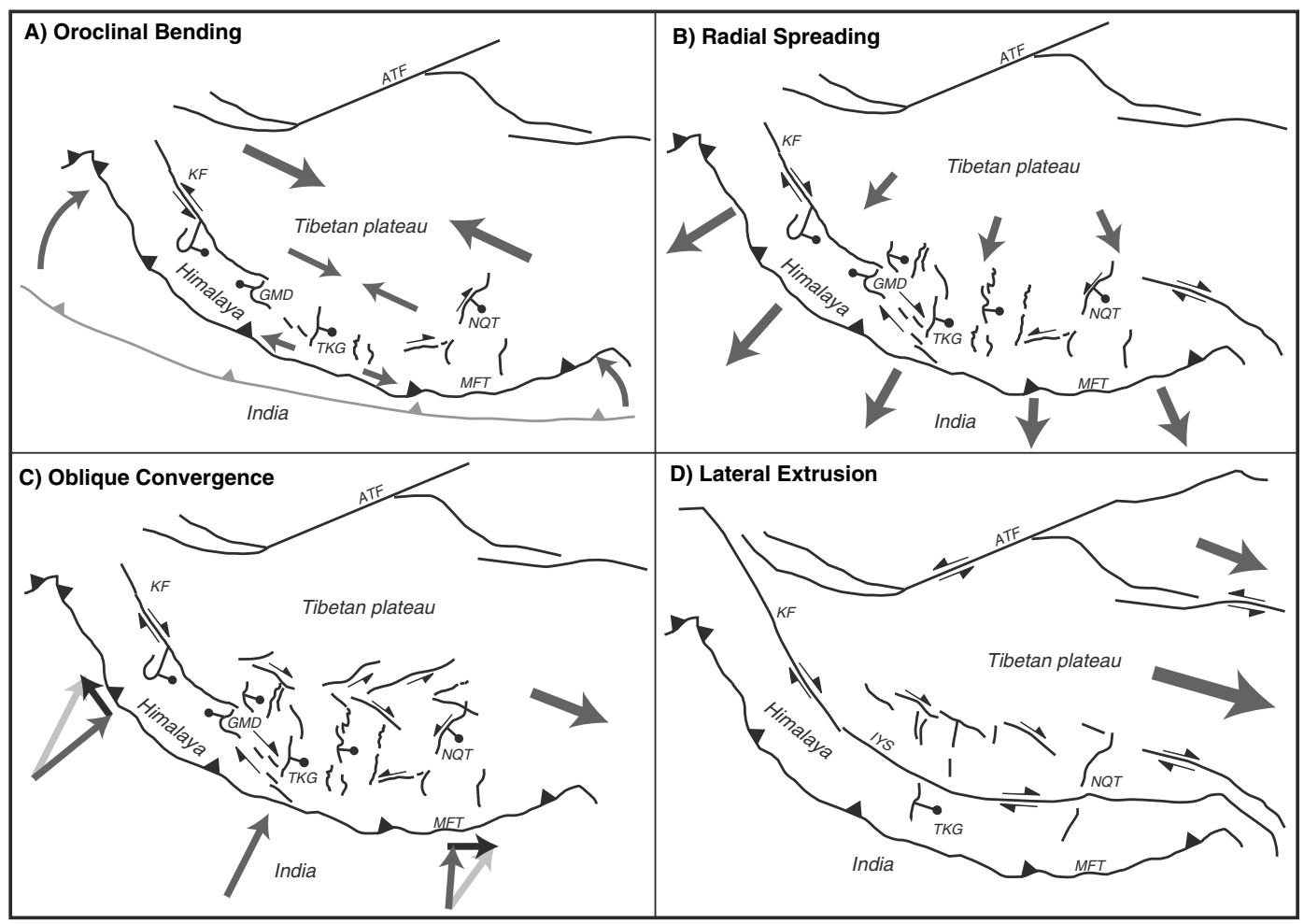

Figure 2. Models of Himalayan and south Tibetan kinematic development. (a) Oroclinal bending, (b) radial spreading, (c) oblique convergence, and (d) lateral extrusion. ATF - Altyn Tagh fault; KF - Karakoram fault; GMD - Gurla Mandhata detachment; IYS - Indus-Yalu Suture; MFT - Main Frontal Thrust; NQT - Nyainqentanglha Shan; TKG - Takkhola Graben. Figure modified from Styron et al. [2011].

extends eastward and along the Indus-Yalu suture zone, thereby bypassing the Himalayan thrust belt. Alternatively, oblique convergence interprets that a significant component of fault slip is fed southward into the Himalayan thrust wedge along the Gurla Mandhata detachment (GMD) fault. To evaluate the validity of these structural interpretations, we conducted an investigation of the thermal history of the GMD footwall aimed at estimating fault slip magnitude, slip rate, and age of initiation for the GMD. We then compare our results with the magnitude of fault slip, fault slip rate, and the timing of initiation for the KF system.

\section{Geologic Setting}

The KF and the IYS zone play important roles in models explaining the tectonic evolution of the Tibet-Himalayan orogen. The dextral KF broadly coincides with the western boundary of the Tibetan plateau, from the Pamirs in the north to the Mt. Kailas region in the south. Previous work has shown that the KF is a northwest-striking dextral fault with $\sim 149-167 \mathrm{~km}$ of offset along the northern fault segment based on offset reconstructions of the Aghil Formation [Robinson, 2009]. Along the central section, some have argued for $400-280 \mathrm{~km}$ of slip based on the offset reconstruction of the IYS zone [Lacassin et al., 2004; Valli et al., 2007, 2008]. Others have argued for 150-120 km of slip along its central section based on the offset reconstruction of the Indus river as well as offset granite bodies [Searle, 1996; Searle et al., 1998]. Along the southern section of the KF, $65 \mathrm{~km}$ of slip is estimated based on the offset reconstruction of the south Kailas thrust system [Murphy et al., 2000], and 50-35 km based on retrodeforming the Gar basin [Sanchez et al., 2010] and correlation of offset granitic bodies [Wang et al., 2012; Wang et al., 2013]. The central segment of the KF is estimated to have initiated $\sim 16$ Ma ago [Searle et al., 1998] and to have propagated southward, where it offsets the south Kailas thrust system, which is interpreted to have last moved between 18 and $10 \mathrm{Ma}$ [Yin et al., 1999; Zhang et al., 2011]. This study focuses on the southern fault segment of the KF where considerable debate exists on the kinematic interplay between the KF and the Xiao Gurla segment of the GMD system (Figures 2 and 5).

The IYS zone locally marks the boundary between the Lhasa terrane and the Tethyan Himalaya (Figure 1). Where mapped, the IYS zone is delineated by the north-directed Great Counter Thrust [Yin et al., 1999; Murphy 
et al., 2009, 2010; Sanchez et al., 2013]. The IYS developed from the collision between the Indian and Eurasian plates and has also been interpreted to be active today as a dextral structure accommodating the lateral extrusion of Tibetan crust [e.g., Chevalier et al., 2012; Tapponnier et al., 1982].

\subsection{Geology of Gurla Mandhata}

The GMD fault lies south of the IYS zone. Murphy et al. [2002] described it as a series of west dipping, moderateto low-angle $\left(22-45^{\circ}\right)$ normal faults bounding the western and northern flanks of the Gurla Mandhata massif. Estimates of fault slip range between 35 and $66 \mathrm{~km}$ for the area along the northern and central sections of the fault [Murphy et al., 2002] and $\sim 35 \mathrm{~km}$ of slip along the southern section of the massif [Murphy and Copeland 2005]. ${ }^{40} \mathrm{Ar} /{ }^{39} \mathrm{Ar}$ thermochronology of biotite and muscovite is consistent with exhumation of its footwall ca. $9 \mathrm{Ma}$ ago [Murphy et al., 2002; Murphy and Copeland, 2005].

A series of en-echelon normal faults cut the Pulan Basin, located in the immediate hanging wall of the GMD. These faults are primarily located on the eastern side of the Pulan basin (Figure 3), but previous studies have locally observed recently active faults along the western boundary [Murphy et al., 2002; Murphy and Copeland, 2005; Murphy and Burgess, 2006; Chevalier et al., 2012]. The active, west dipping, high-angle normal faults have a mean slip direction of $274^{\circ} \pm 10^{\circ}$ and a maximum throw of $200 \mathrm{~m}$ based on reconstructing offset MiocenePliocene sedimentary rocks [Murphy et al., 2002]. The high angle faults are interpreted to sole into the Gurla Mandhata detachment system at depth (Figure 4a). At the southernmost segment of these high-angle faults, the GMD strikes eastward and transfers slip into the dextral Humla Fault (Figure 3).

\subsection{Lithologic Units}

The hanging wall of the GMD is composed of eight units - these include: (1) Kiogar Ophiolites (op), (2) Tethyan sequence (ts) rocks, (3) Cretaceous-Tertiary volcanic rocks (K-Tv), (4) Cretaceous-Tertiary granite (K-T), (5) Kailas sequence (ks) rocks, (6) Mesozoic group (Mv) rocks, (7) Pulan Basin strata (Tcg), and (8) Pulan basin Quaternary alluvium (Qtr). The footwall of the GMD is comprised of five units - these include: (1) Migmatite (mig), (2) gneiss (gn), (3) Greater Himalayan sequence (ghs) rocks, (4) Xiao Gurla sequence (xg) rocks, and (5) Leucogranite (gr).

2.3.1. Hanging Wall Units

The Kiogar Ophiolite sequence (op) is the oldest unit within the hanging wall rocks. The ophilitic rocks are mainly composed of norite, dunite, and harzburgite [Miller et al., 1999; Murphy et al., 2002]. Tethyan sequence (ts) is exposed in the western edge of the field area and is a $>9 \mathrm{~km}$ thick section of Upper Proterozoic through Lower Cretaceous sandstone, shale, and limestone [Murphy and Yin, 2003]. This sequence is repeated by a series of north dipping thrust faults (Figure 3 ).

The Pulan basin strata are separated into two sequences: the older Pulan basin strata (Tcg) and the younger Pulan basin strata (Qtr). The older Pulan basin strata (Tcg) are composed of sandstones, shales, and conglomerates confined mainly to the southern half of the Pulan basin [Murphy et al., 2002]. The younger strata (Qtr) are composed of clasts derived mainly from the footwall of the Gurla Mandhata detachment fault (Figure 3) [Murphy et al., 2002].

\subsubsection{Footwall Units}

Migmatitic gneiss (mig) is the structurally lowest unit in the footwall of the GMD (Figure 4). The rocks that make up the migmatite are banded gneisses with biotite-rich layers and numerous leucosomes. A penetrative mylonitic fabric with the same shear sense as the GMD is observed throughout the unit [Murphy et al., 2002]. Geochemical analysis by Murphy [2007] found that Nd and Sr isotope ratios of portions of the migmatite are consistent with a Lesser Himalayan sequence (Ihs) protolith.

Within the footwall, but structurally above the migma-titic gneiss (mig) are quartzofeldspathic gneisses and biotite schists (gn) (Figure 4) [Murphy et al., 2002]. Penetrative ductile shear fabrics within these rocks are consistent with top-to-the-west shear sense $\left(280 \pm 4^{\circ}\right)$ [Murphy et al., 2002]. Leucogranite dikes intrude the gneiss (gn), and the dikes are sheared with the same top-to-the-west shear sense as the ductile fabrics. Crosscutting relationships and shear sense indicators show that the dikes were likely emplaced during simple shear [Murphy et al., 2002]. Geochemical analyses indicate the gneisses have the same $\mathrm{Nd}$ and $\mathrm{Sr}$ isotopic signature as the Greater Himalayan sequence (ghs) [Murphy, 2007].

The Greater Himalayan sequence (ghs) makes up the structurally highest unit of the Gurla Mandhata massif (Figure 4) The majority of these rocks are highly deformed marbles and schists with top-to-the-west shear 


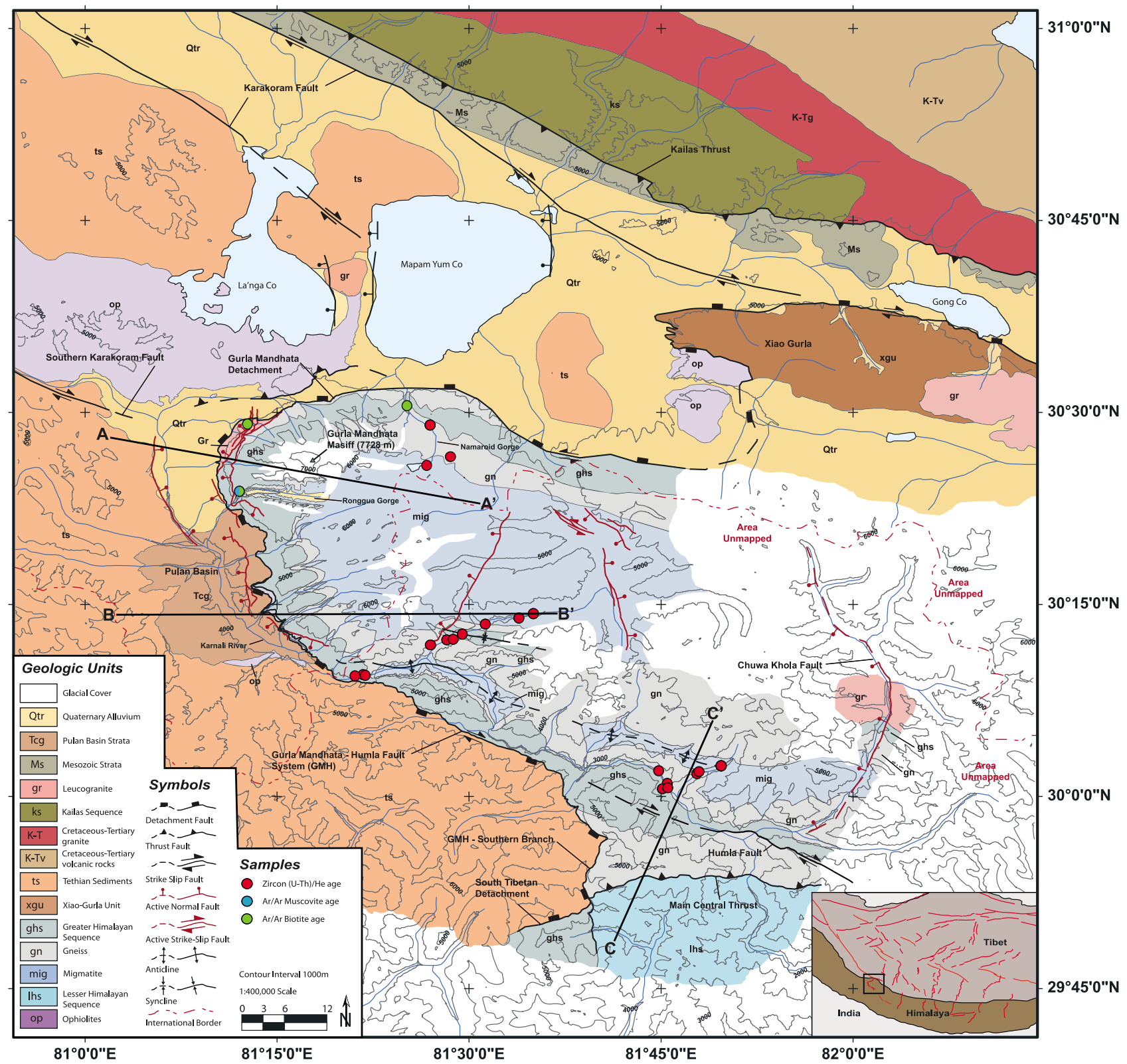

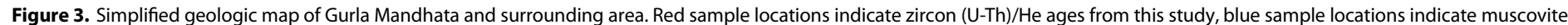

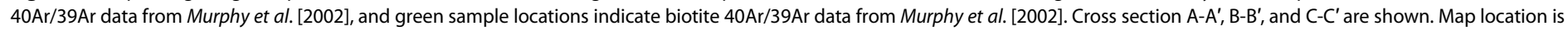
shown on the inset in the bottom right corner. Modified from Murphy et al. [2002], Murphy and Copeland [2005], Murphy and Burgess [2006], and Pullen et al. [2011].

sense, along with extensive leucogranite intrusions also displaying the same shear sense, suggesting synkinematic emplacement [Murphy et al., 2002].

The Xiao Gurla sequence $(\mathrm{xg})$ is composed of metamorphosed Tethyan sedimentary sequence (ts) rocks. Detrital $\mathrm{U} / \mathrm{Pb}$ zircon analysis shows the Xiao Gurla sequence $(\mathrm{xg})$ has the same detrital signature and lithology as the local Tethyan sedimentary sequence (ts) [Pullen et al., 2011]. Leucogranite dikes similar to the Gurla Mandhata footwall also intrude the Tethyan rocks.

The leucogranite ( $\mathrm{gr}$ ) dikes compose the youngest footwall unit. This unit makes up $10-20 \%$ of the footwall and intrudes all of the footwall units [Murphy et al., 2002; Murphy and Copeland, 2005; Pullen et al., 2011]. It is commonly observed as $2 \mathrm{~m}$ thick dikes and sills with a similar sense of shear as the main faulting direction (top to the west), which suggests synkinematic emplacement [Murphy et al., 2002; Murphy and Copeland, 2005; Pullen et al., 2011]. Pullen et al. [2011] dated seven zircons from the footwall of the GMD, with a mean U- 

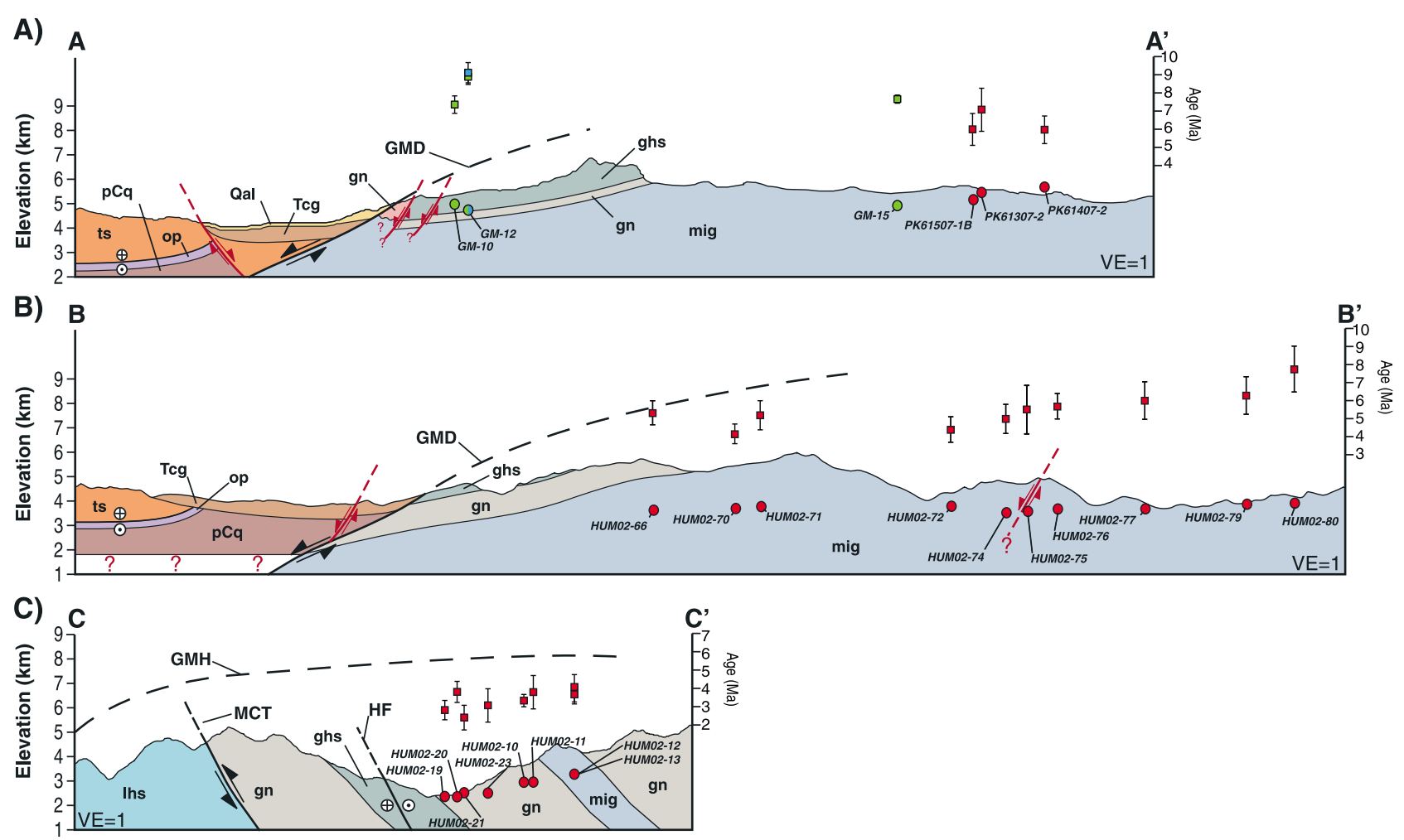

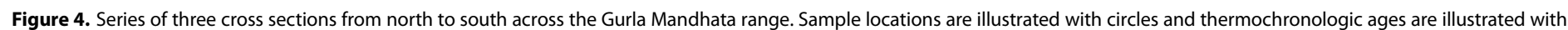

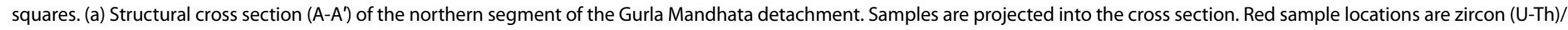

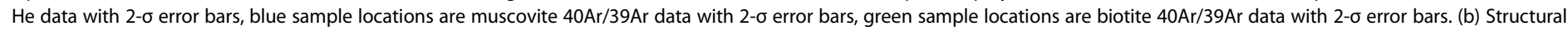

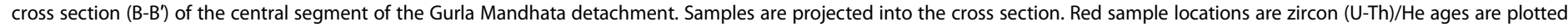

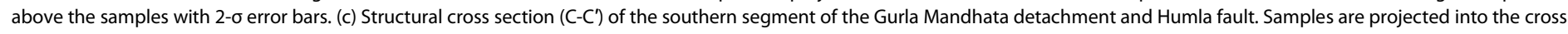

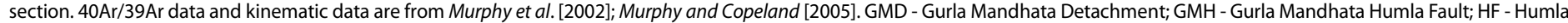
Fault; MCT - Main Central Thrust.

$\mathrm{Pb}$ age of $18.6 \pm 0.9 \mathrm{Ma}$, along with seven zircon grains from the Xiao Gurla leucogranite sequence yielding an average $\mathrm{U} / \mathrm{Pb}$ age of $19.5 \pm 1.5 \mathrm{Ma}$. These samples are indistinguishable within error. They have crystallization ages older than the interpreted initiation age of the GMD of about 12 Ma [Murphy et al., 2002] suggesting that some intrusions may be coeval with deformation and others may predate deformation [Murphy et al., 2002; Murphy and Copeland, 2005; Pullen et al., 2011].

\section{Methods}

\subsection{Zircon (U-Th)/He Thermochronology}

(U-Th)/He low-temperature thermochronometry is a well-established technique for examining the cooling history of exhumed footwalls of normal faults [Stockli, 2005]. For this study, we use zircon because it is relatively abundant in the footwall rock, and it has a partial retention zone that can track upper crustal processes between 175 and $190^{\circ} \mathrm{C}$ [Reiners, 2005; Stockli, 2005]. The samples analyzed here are described based on their relationship to the three transects described beginning in section 3.1.3.

\subsubsection{Sample Processing}

All samples were processed at the University of Kansas, Isotope Geochemistry Laboratory (IGL) using standard mineral separation techniques. For each sample, 3-8 zircon grains were analyzed using the methods described in Wolfe and Stockli [2010]. Individual zircon grains were packed in platinum packets and degassed under highvacuum conditions. The degassed grains were removed from the platinum packets, dissolved, and the concentrations of the He parent isotopes of $U$, Th, and Sm were determined by isotope dilution on a Thermo Scientific Element 2 inductively coupled plasma mass spectrometer (ICP-MS). 


\subsubsection{Samples}

Samples were collected along three footwall transects subparallel to the slip direction of the GMD (Figure 3). Each transect is between 25 and $35 \mathrm{~km}$ in length across the footwall, with a 500-1000 m mean elevation change, and consists of 3-10 bedrock samples. Below we describe each transect from north to south.

\subsubsection{Northern Transect}

The northern transect consists of three (U-Th)/He thermochronometric samples collected along the Namarodi Valley northeast of the Gurla Mandhata peak (Figure 3). Two of the three samples were collected along the valley walls from exposed bedrock, while the third sample was collected from a ridge just east of the main valley (Figure 3). A mean elevation gain of $\sim 500 \mathrm{~m}$ separates the lowest sample from the highest (A$A^{\prime}$; Figure 4a).

\subsubsection{Central Transect}

The central transect is $35 \mathrm{~km}$ long and starts at the southern extent of the Pulan Basin (corresponding with cross section $B-B^{\prime}$, Figure $4 b$ ) and continues up the Karnali tributary to the northeast into the core of the range (Figure 3). Thermochronometric samples were collected along the river valley floor or along the valley wall from exposed bedrock and spans $\sim 700 \mathrm{~m}$ in elevation change. Ten samples were collected from the transect, representing all four of the footwall units (Table 1).

\subsubsection{Southern Transect}

The southern transect starts in the Karnali River valley close to the northwest termination of the Humla Fault, corresponding with cross section C-C' (Figure 4c). The sample transect continues to the northeast, up a tributary gaining $\sim 800 \mathrm{~m}$ in elevation and comprises eight samples from three of the four footwall units (Table 1).

\subsubsection{Results}

For this study, 21 samples were analyzed (3-7 aliquots each), with weighted averaged ages ranging from $2.6 \pm 0.7$ to $8.0 \pm 1.3 \mathrm{Ma}$ (Table 1). The sample transects show (U-Th)/He ages become progressively older eastward into the footwall and with an increase in elevation (Figure 4). The northern transect consists of three samples with a minimum age of $6.1 \pm 0.8 \mathrm{Ma}$ and a maximum age of $7.2 \pm 1.2 \mathrm{Ma}$. The sample ages increase with elevation. The central transect consists of 10 samples (3-7 aliquots) ranging in age from $4.5 \pm 1.1 \mathrm{Ma}$ to $8.0 \pm 1.3$ Ma. The age of the samples increase gradually into the GMD footwall and with increasing elevation (Table 1). The southern transect consists of eight samples (4-5 aliquots) ranging from $2.6 \pm 0.7 \mathrm{Ma}$ to $4.2 \pm 0.8 \mathrm{Ma}$ in age, with the sample age increasing with elevation.

Comparing the three sample transects reveals an overall decrease in age to the south. This could be explained by a sampling bias, as the northern and southern transects do not intersect with the range front. Potential bias aside, with the exception of the last sample in the central transect, there is a southward decrease in oldest age between the three transects. At the eastern end of the central transect, there is a small age jump from $6.5 \mathrm{Ma}$ to $8.0 \mathrm{Ma}$ (Figure $4 \mathrm{~b}$ and Table 1). This increase is not due to a large elevation gain, suggesting a possible structural control. In addition to this trend, the extremely young ages across the entirety of the range suggest that the range has been exhumed quickly over most of its development. Although there are extensive intrusions throughout the Gurla Mandhata range [Murphy et al., 2002; Murphy and Copeland, 2005] and nearby ranges [Pullen et al., 2011], published zircon U-Pb crystallization ages for the GMD and nearby ranges are consistent with granitic intrusions emplaced prior to $18 \mathrm{Ma}$ [Pullen et al., 2011].

\section{Thermokinematic Modeling}

In order to evaluate fault initiation, fault slip rate, and duration, we conducted thermokinematic modeling of our low temperature thermochronologic data from the GMD footwall rocks. Because the sampling transects across the GMD are horizontal and no samples have more than one thermochronometric age, conventional modeling software such as HeFTy or HeMP is insufficient to determine the cooling history of the GMD. For this reason, the data for the GMD are modeled in the finite element software package Pecube. In the following, we briefly describe the Pecube software followed by the thermal parameters and model assumptions used in this study.

\subsection{Pecube}

The zircon (U-Th)/He data were modeled using Pecube finite element software [Braun, 2003]. Pecube incorporates internal heating, isotherm advection, and a wide variety of thermochronometers [Braun, 2003]. Due to the spatial range of samples and the variability of the fault plane geometry, each individual transect was modeled 


\begin{tabular}{|c|c|c|c|c|c|c|c|c|}
\hline 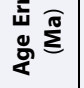 & \multicolumn{2}{|c|}{$\tilde{m}$} & à̀ & 㐫 & in & กู่ & $\stackrel{\circ}{\circ}$ & 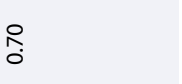 \\
\hline 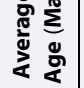 & & fo & นิ & ปั & مิ & ஸे & Бे & กُ \\
\hline : & 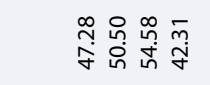 & 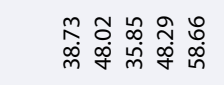 & 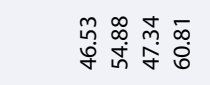 & 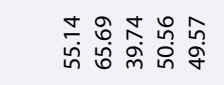 & 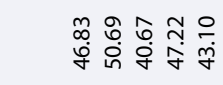 & 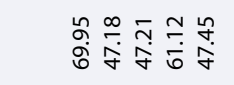 & 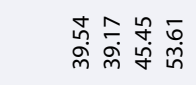 & 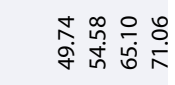 \\
\hline$\ddot{\leftarrow}$ & 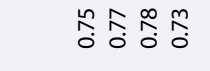 & 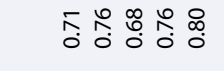 & 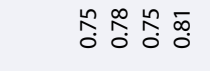 & 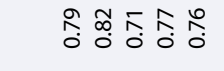 & 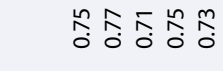 & 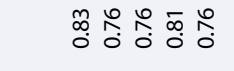 & 중종 & $\begin{array}{c}\hat{A} \\
\hat{0} \\
0\end{array}$ \\
\hline है के & 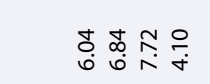 & 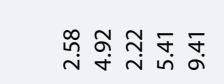 & 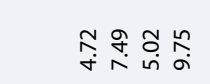 & 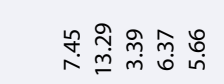 & 䏍 & 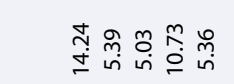 & $\underset{\sim}{\infty} \underset{\sim}{\stackrel{n}{\sim}} \stackrel{\circ}{\stackrel{\sim}{\leftarrow}}$ & 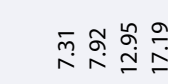 \\
\hline 일 & 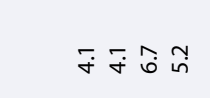 & 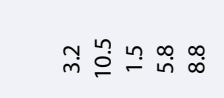 & $\stackrel{\infty}{+} \stackrel{\infty}{\sim} \underset{\infty}{\infty} \stackrel{\mathfrak{n}}{=}$ & 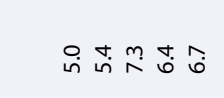 & 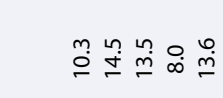 & 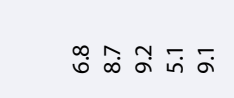 & 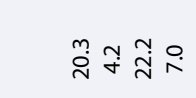 & 苍 \\
\hline 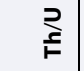 & 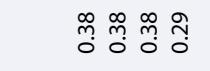 & 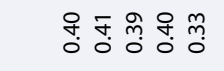 & 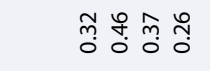 & 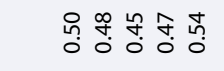 & 융 정융 & 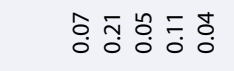 & 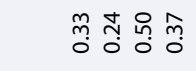 & 옹 \\
\hline$\stackrel{0}{\Sigma}$ & 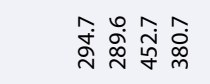 & 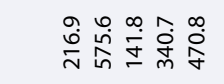 & 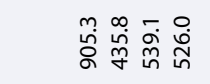 & 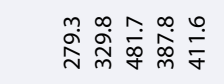 & 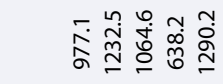 & 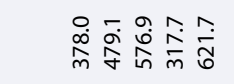 & 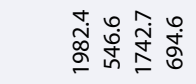 & 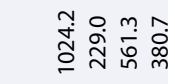 \\
\hline 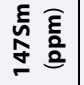 & 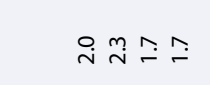 & 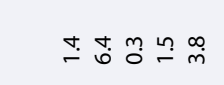 & 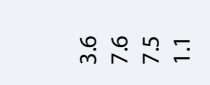 & & $\tilde{N} \stackrel{m}{\sim} \dot{N} \bar{m} \hat{N}$ & ñ. & $\cong \stackrel{\infty}{+} \bar{i} \hat{i}$ & กิธ่์ ó. \\
\hline$\varepsilon \overline{\frac{\bar{g}}{2}}$ & 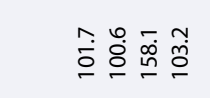 & 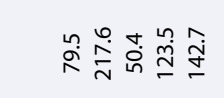 & 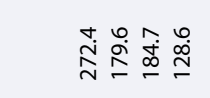 & 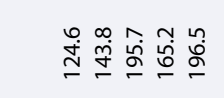 & 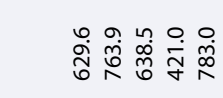 & 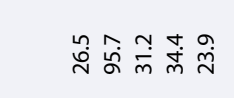 & 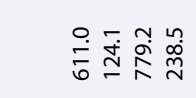 & 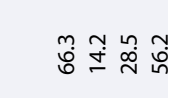 \\
\hline 工 $\overline{\frac{\mathrm{g}}{g}}$ & 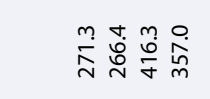 & 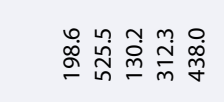 & 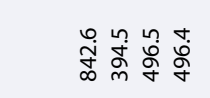 & 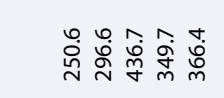 & 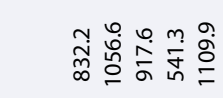 & 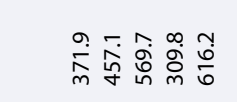 & 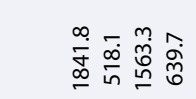 & 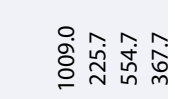 \\
\hline$\stackrel{\Sigma}{\dot{z}} \stackrel{\pi}{\Sigma}$ & 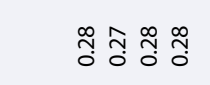 & $\bar{m}$ o & 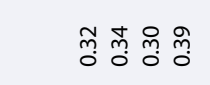 & 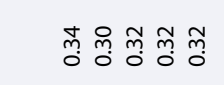 & 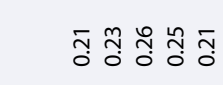 & 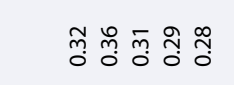 & 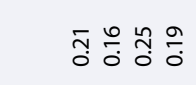 & 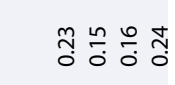 \\
\hline$\stackrel{9}{\frac{\pi}{2}}$ & $\stackrel{q}{m} \underset{m}{m} \stackrel{\infty}{\stackrel{f}{m}} \stackrel{g}{m}$ & 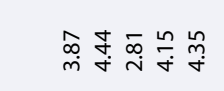 & $\underset{+}{\sim} \bar{\sim} \underset{\sim}{\sim} \underset{m}{\stackrel{\infty}{\infty}}$ & 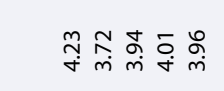 & 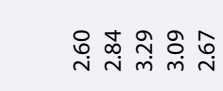 & 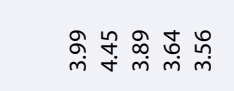 & 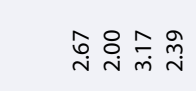 & 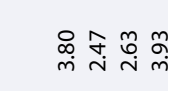 \\
\hline 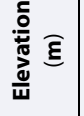 & 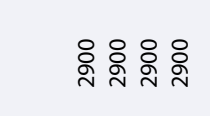 & 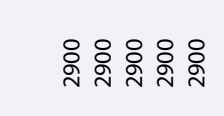 & 家总总 & $\frac{8}{m} \frac{8}{m} \frac{8}{m} \frac{8}{m} \frac{8}{m}$ & 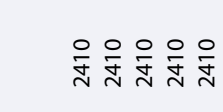 & 䧛号 帝 & 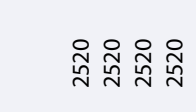 & 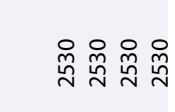 \\
\hline 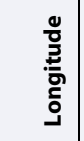 & 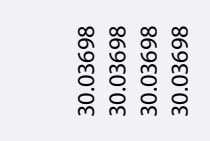 & 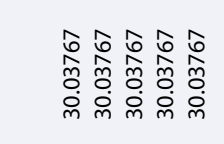 & 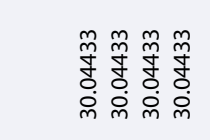 & 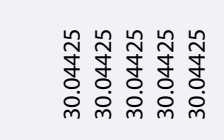 & 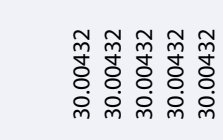 & 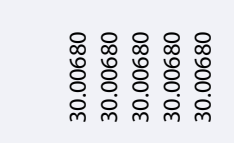 & 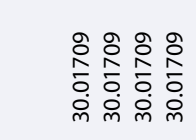 & 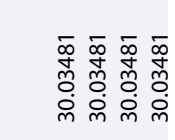 \\
\hline 苋 & 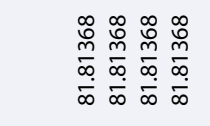 & 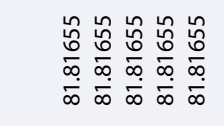 & 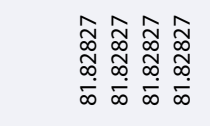 & 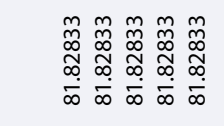 & 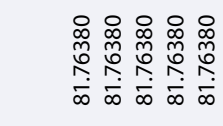 & 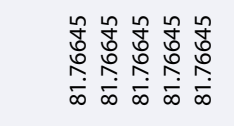 & 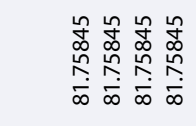 & 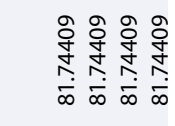 \\
\hline 产 & 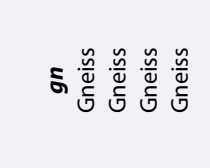 & 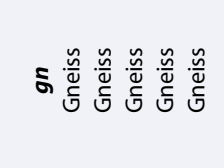 & 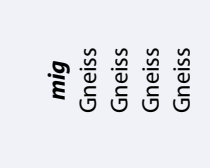 & 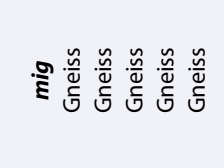 & 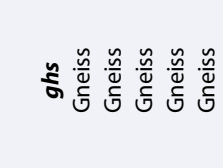 & 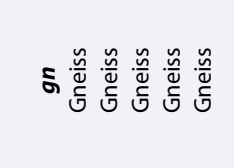 & 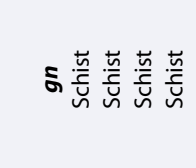 & 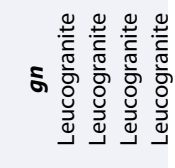 \\
\hline 嵒 & 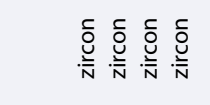 & 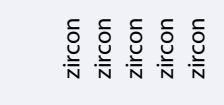 & 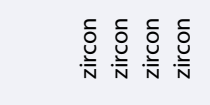 & 总总总 & 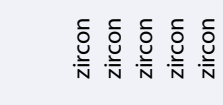 & 总号 & 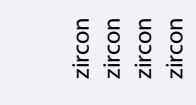 & 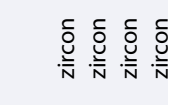 \\
\hline $\begin{array}{l}\frac{0}{0} \\
\frac{2}{5} \\
\text { ñ }\end{array}$ & 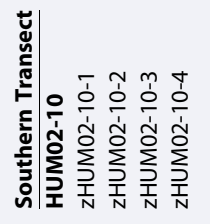 & 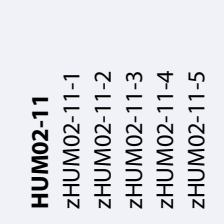 & 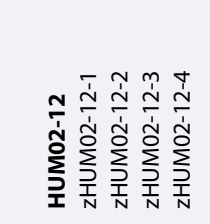 & 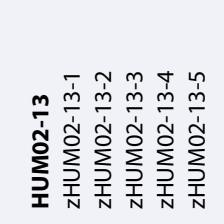 & 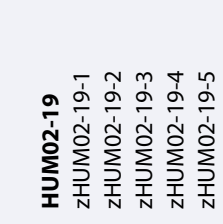 & 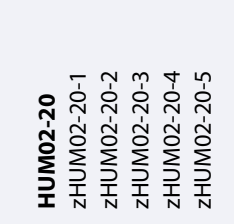 & 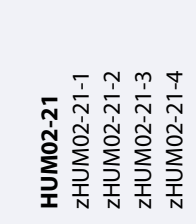 & 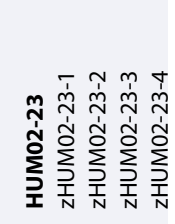 \\
\hline
\end{tabular}




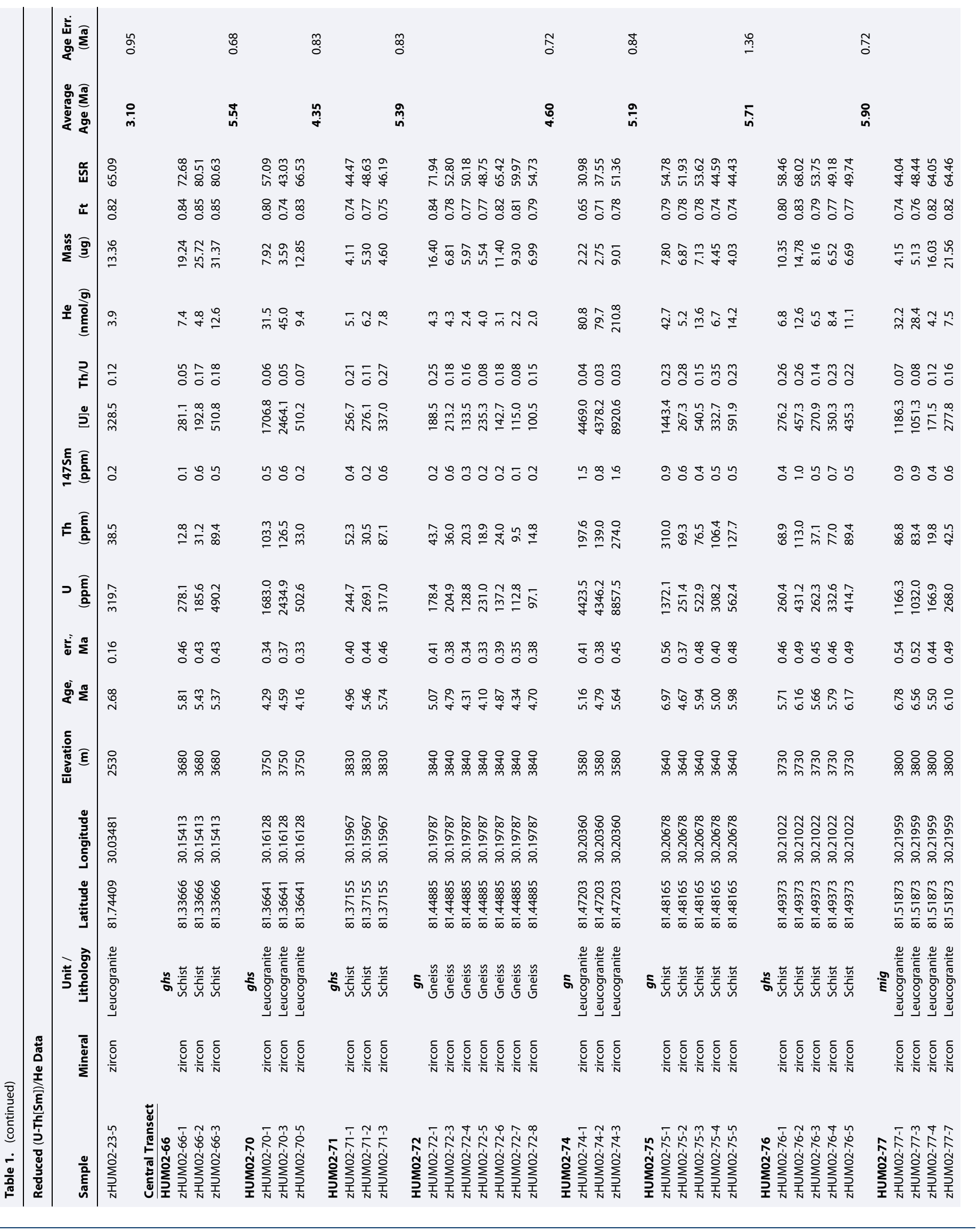




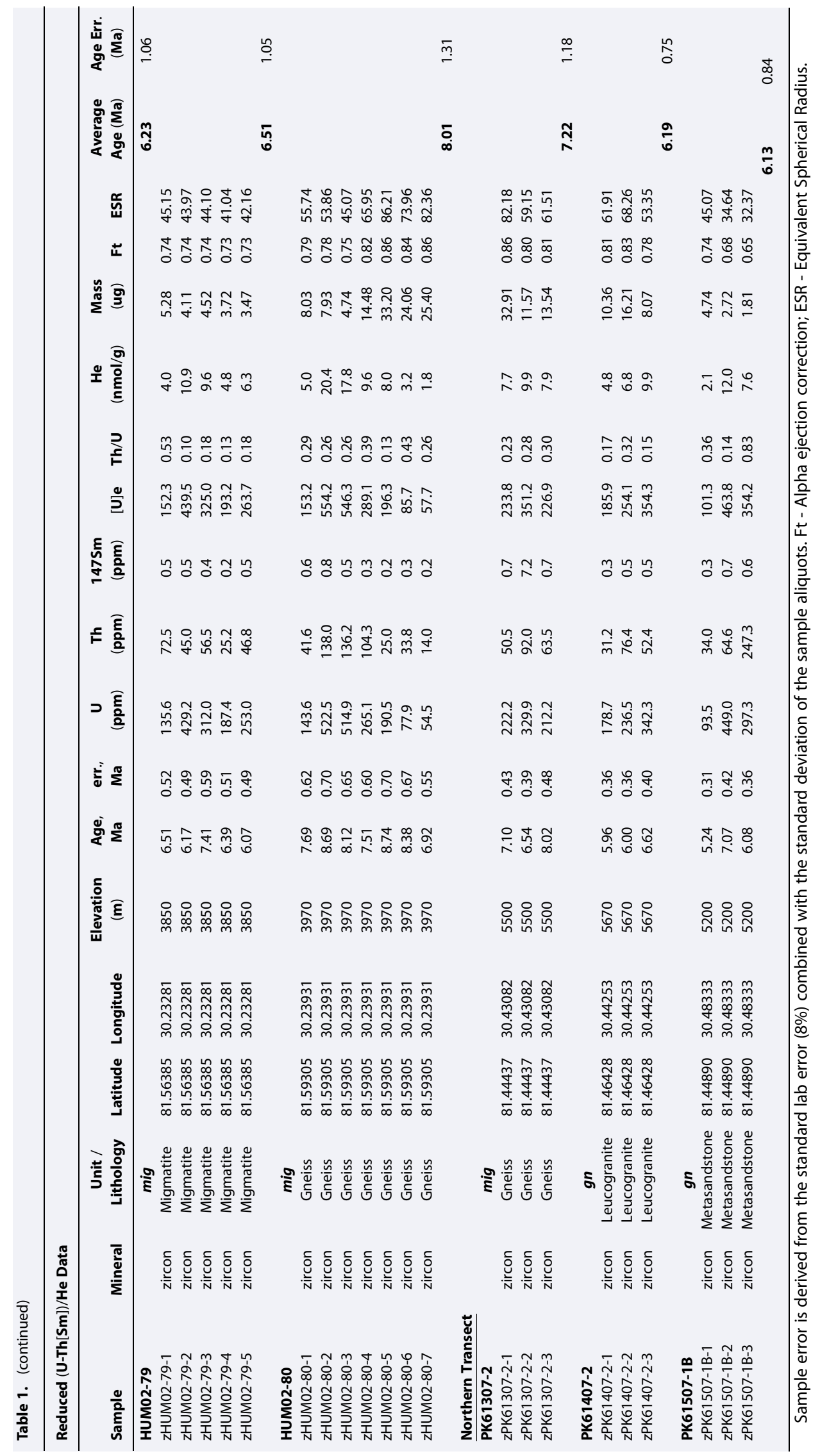




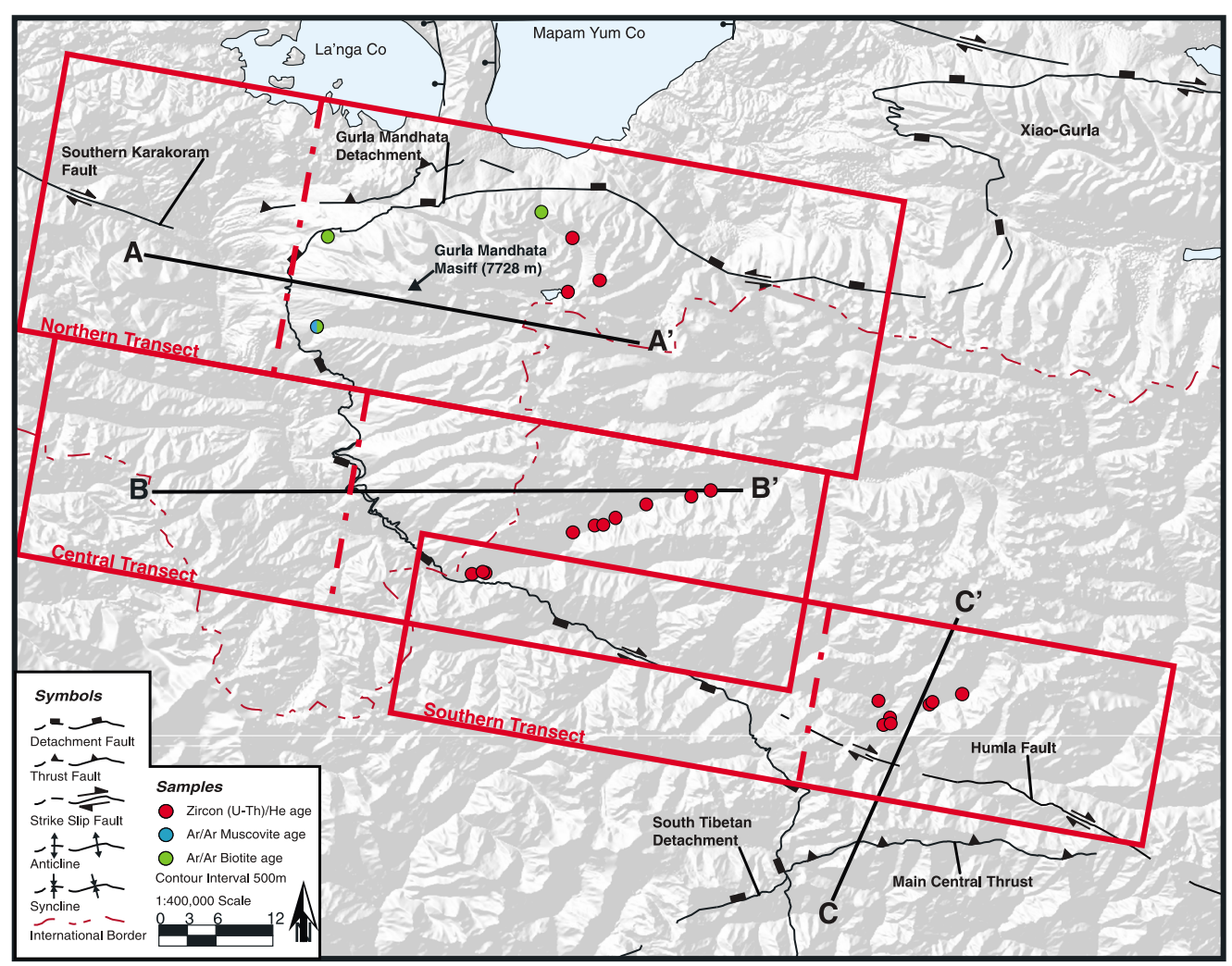

Figure 5. Hill shade map of the GMD with the major faults shown for reference. Red squares show the location of each of the three Pecube models (northern, central, and southern transects) and their relation to the samples (Red - zircon (U-Th)/He; Blue - muscovite 40Ar/39Ar; Green - biotite $40 \mathrm{Ar} / 39 \mathrm{Ar}$ ) and cross sections $\left(\mathrm{A}-\mathrm{A}^{\prime}, \mathrm{B}-\mathrm{B}^{\prime}\right.$, and $\left.\mathrm{C}-\mathrm{C}^{\prime}\right)$. Each model has been oriented to the mean slip direction $\left(100^{\circ}\right)$. The dashed red lines in each box show the orientation of the modeled detachment fault within each model.

separately (Figure 5). Oriented in the slip direction, the data are ordered as follows: (1) the northern transect, located in the Ronggua Gorge, is composed of two ${ }^{40} \mathrm{Ar} /{ }^{39} \mathrm{Ar}$ muscovite and three ${ }^{40} \mathrm{Ar} /{ }^{39} \mathrm{Ar}$ biotite ages (as published by Murphy et al. [2002]) and represented by three zircon (U-Th)/He samples (composing a vertical transect) (Figure 4a)); (2) the central transect, represented by 10 zircon (U-Th)/He samples (Figure 4b); and (3) the southern transect, located at the inferred intersection of the Humla fault with the GMD, which includes eight zircon (U-Th)/He samples (Figure 4c).

For all transects, the model space is oriented parallel to the average-slip direction $\left(100^{\circ}\right)$ (Figure 5) to better model the path that the samples would have traveled. The Moho depth is $65 \mathrm{~km}$ [Nabelek et al., 2009; Chen et al., 2010], as estimated by seismic Hi-Climb transects, with a base temperature of $800^{\circ} \mathrm{C}$ [Nabelek et al., 2010; Nabelek and Nabelek, 2011]. The internal heating value of $10^{\circ} \mathrm{C} / \mathrm{My}$ is based on radiogenic heat production [Faccenda et al., 2008].

The fault geometry is based on cross sections presented in Murphy et al. [2002]. For the northern and central transects, the model is allowed to initiate between 18 and $9 \mathrm{Ma}$, based on work by Murphy et al. [2002], with a fault slip rate between 1 and $12 \mathrm{~mm} / \mathrm{yr}$. For the southern transect, the model is allowed to initiate between 15 and $7 \mathrm{Ma}$, based on work by Murphy and Copeland [2005], with a fault slip rate of 1-12 mm/yr. To better model the faults, all three transects are allowed to change slip rates once between 14 and $0 \mathrm{Ma}$ to rates between 0 and $12 \mathrm{~mm} / \mathrm{yr}$. The velocity field is calculated by Pecube based on the imposed fault geometry and slip rate. In these models, the hanging wall is held fixed and fault slip results in motion of the footwall only, at the velocity applied to the fault. The antilistric geometry of the detachment (i.e., doming and back-rotation of the footwall at the surface) results in footwall exhumation at depth, with a decrease in exhumation and an increase in horizontal translation as a particle approaches the surface. This detachment geometry is consistent with geologic observations, including the warping of the mylonitic fabric and the rocks exhumed from the greatest depths near to 


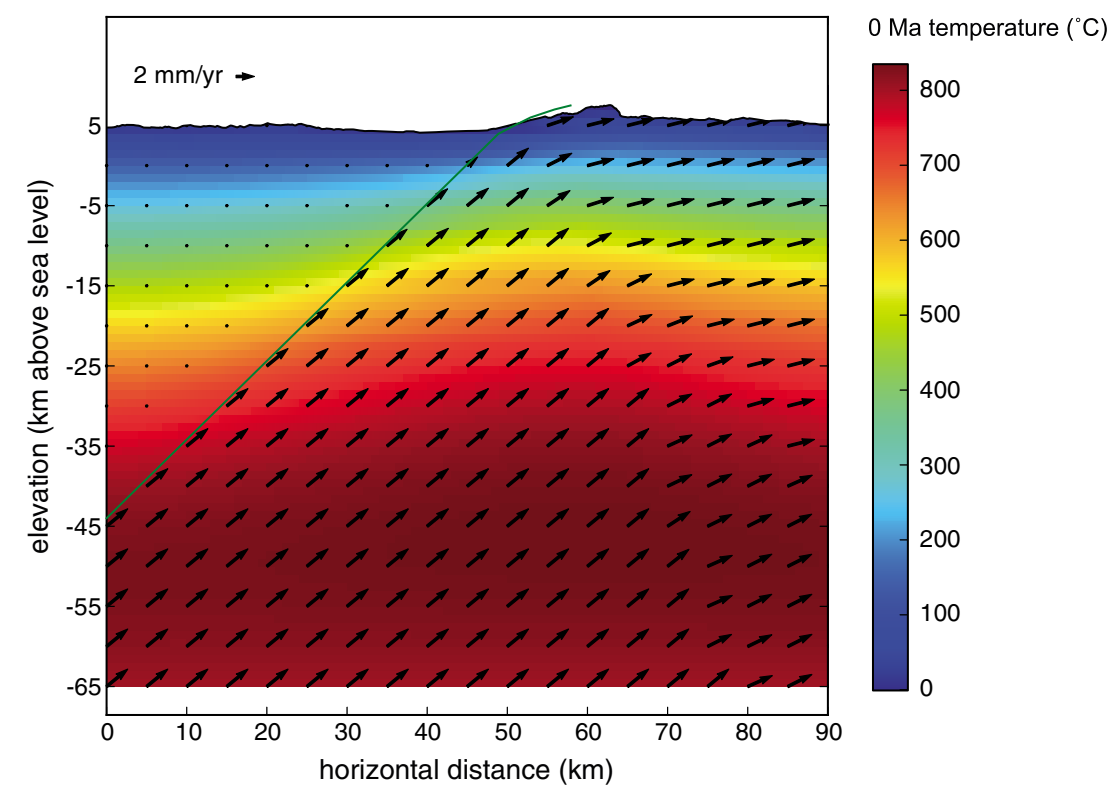

Figure 6. Example velocity field for the North Transect. Black arrows indicate direction and magnitude of footwall velocity relative to the hanging wall. Green line is the Gurla Mandhata Detachment. Background colors represent the temperature field at $0 \mathrm{Ma}$, following a fault history with $5 \mathrm{~mm} / \mathrm{yr}$ slip on the GMD since $14 \mathrm{Ma}$. Note the advection of heat in the footwall.

the detachment, as well as thermochronologic observations of the youngest cooling ages in the footwall closest to the range-bounding detachment.

The equation for the velocity field caused by fault slip is:

$$
V_{o}^{\prime}=V_{o} \frac{\cos \alpha}{\cos \frac{\alpha}{2}}
$$

[Braun et al., 2012]. Where $V_{0^{\prime}}$ is the velocity at a point, $V_{0}$ is the fault slip rate, and $\alpha$ is the interior angle of the bend in the detachment. The mean divergence is not constrained to be zero, but the results approach zero.

Several potential issues arise from complications within the fault system and computational limitations within Pecube: (1) The fault geometry of the GMD is complex and variable. In order to reconcile this complication, we use the mean fault dip for the corresponding fault segment. (2) Limitations within Pecube prevent any change in fault geometry during faulting. For this reason, we are limited to a static fault geometry as observed at the surface and the structural response of the hanging wall [Murphy et al., 2002; Murphy and Copeland, 2005]. (3) Because all faults within the Pecube model have the same strike and along-strike length, any potential influence of the northern Humla Fault along the southern segment cannot be investigated. (4) Pecube limitations require all fault slip to be either normal or thrust, causing any strike-slip component to be negated. (5) Topography is considered to be steady state. Although these limitations are not specifically addressed in this work, we believe the modeling using Pecube is nonetheless sufficient to capture the Neogene thermal evolution of the GMD footwall.

\subsection{Northern Transect}

The northern transect corresponds to cross section A-A' (Figure 4a). The model space extends well beyond the sample locations to avoid edge effects. The model is oriented to $280^{\circ}$, which is the general fault slip direction [Murphy et al., 2002; Murphy and Copeland 2005]. The fault strikes $010^{\circ}$ with a west dip of $30^{\circ}$. The shape of the fault plane is depicted in Figure 6. We include two muscovite samples $\left({ }^{40} \mathrm{Ar} /{ }^{39} \mathrm{Ar}\right.$ cooling ages), one biotite sample $\left({ }^{40} \mathrm{Ar} /{ }^{39} \mathrm{Ar}\right.$ cooling age), all of which are from the Ronggua Gorge, and two biotite samples $\left({ }^{40} \mathrm{Ar} /{ }^{39} \mathrm{Ar}\right.$ cooling age) from the Namarodi Valley [Murphy et al., 2002]. Early testing of model parameters show that a curved geometry projected above the surface is needed to reproduce the observed age-elevation trend. The domal fault geometry is also constrained by field observations, and fault traces bounding the large triangular 
Table 2. Table of Variables Tested in Each of the Three Transects and the Parameters Used in the Basic Pecube Model

\section{PeCube Transect}

Parameters

\begin{tabular}{|c|c|c|c|}
\hline Northern Transect & Range & Units & References \\
\hline Initiation Age & $18-9$ & $\mathrm{Ma}$ & [Murphy et al., 2002] \\
\hline Acceleration Age & $14-0$ & $\mathrm{Ma}$ & \\
\hline Initial Rate & $1-12$ & $\mathrm{~mm} / \mathrm{yr}$ & \\
\hline Postacceleration Rate & $0-12$ & $\mathrm{~mm} / \mathrm{yr}$ & \\
\hline Fault Slip & $14-62$ & $\mathrm{~km}$ & \\
\hline \multicolumn{4}{|l|}{ Central Transect } \\
\hline Initiation Age & $18-9$ & $\mathrm{Ma}$ & [Murphy et al., 2002] \\
\hline Acceleration Age & $14-0$ & Ma & \\
\hline Initial Rate & $1-12$ & $\mathrm{~mm} / \mathrm{yr}$ & \\
\hline Postacceleration Rate & $0-12$ & $\mathrm{~mm} / \mathrm{yr}$ & \\
\hline Fault Slip & $14-62$ & $\mathrm{~km}$ & \\
\hline \multicolumn{4}{|l|}{ Southern Transect } \\
\hline Initiation Age & $15-7$ & Ma & $\begin{array}{l}\text { [Murphy and Copeland, } \\
\text { 2005] }\end{array}$ \\
\hline Acceleration Age & $14-0$ & $\mathrm{Ma}$ & \\
\hline Initial Rate & $1-12$ & $\mathrm{~mm} / \mathrm{yr}$ & \\
\hline Postacceleration Rate & $0-12$ & $\mathrm{~mm} / \mathrm{yr}$ & \\
\hline Fault Slip & $0-62$ & $\mathrm{~km}$ & \\
\hline \multicolumn{4}{|c|}{ PeCube Model Parameters } \\
\hline Fault Dip & 30 & Degrees & $\begin{array}{c}\text { [Murphy et al., 2002; } \\
\text { Murphy and } \\
\text { Copeland, 2005] }\end{array}$ \\
\hline Moho Temperature & 800 & ${ }^{\circ} \mathrm{C}$ & [Nabelek et al., 2010] \\
\hline Radiogenic Heating & 10 & ${ }^{\circ} \mathrm{C} / \mathrm{Myr}$ & [Faccenda et al., 2008] \\
\hline Model Thickness & 65 & $\mathrm{~km}$ & $\begin{array}{l}\text { [Nabelek et al., 2009; } \\
\quad \text { Chen et al., 2010] }\end{array}$ \\
\hline Skipping Facotor & 15 & & \\
\hline Thermal Diffusivity & 25 & $\mathrm{~km}^{2} / \mathrm{Myr}$ & \\
\hline Density & 2700 & $\mathrm{~kg} / \mathrm{m}^{3}$ & $\begin{array}{c}\text { Average granite values } \\
\text { assumed }\end{array}$ \\
\hline Heat Capacity & 224.607 & $\mathrm{~J} / \mathrm{Mol}^{*} \mathrm{~K}$ & $\begin{array}{c}\text { Average granite values } \\
\text { assumed }\end{array}$ \\
\hline
\end{tabular}

facets are observed on the entire western flank of the range. The domal geometry of the footwall is also observed for other large extensional systems in Tibet [e.g., Robinson et al., 2010; Styron et al., 2013].

Based on these initial model parameters, a total of 12,000 models were possible, each with a unique combination of fault history parameters. This set of possibilities was filtered using a resolvable range of possible slip magnitude values (14-62 $\mathrm{km}$ ) based on field relationships and cross-section reconstructions [Murphy et al., 2002], described in Styron et al. [2013]. This filtering reduced the total number of possible runs to $\sim 6700$ models. (See Table 2 for model parameters.) These initial models were then run on Pecube v.3, in parallel on Amazon's EC2 servers through the PiCloud service (www.picloud.com). See Styron et al. [2013] for modeling workflow and Python codes. The initial results show that 45 of 6700 models fit 4 out of 7 data points, and 2 of 6700 models fit 5 out of 7 data points. No models were able to reproduce all 10 data points. None of the models were able to reproduce the GM-12 muscovite age within 2- $\sigma$

(Figure 7a), and most of the modeled ages were found to be younger than GM-12. About a third (34\%) of the

models were able to reproduce the sample GM-12 biotite age (Figure 7a). Only $23 \%$ of the models were able to reproduce sample HUM02-15 within 2- $\sigma$.

Possible initiation ages for faulting range between 16 and $9 \mathrm{Ma}$, with a mean of $11 \mathrm{Ma}$ and a mode of $9 \mathrm{Ma}$ (dark gray; Figure 8a). A large percentage of these best fit models (81\%) predict fault initiation between 12 and $9 \mathrm{Ma}$ with a small percentage (19\%) predicting an older fault initiation age between 16 and $13 \mathrm{Ma}$.

The initial fault slip rates range between 1 and $12 \mathrm{~mm} / \mathrm{yr}$, with a mean of $7 \mathrm{~mm} / \mathrm{yr}$ and a mode of $8 \mathrm{~mm} / \mathrm{yr}$ (dark gray in Figure $8 \mathrm{~b}$ ). The largest group of models (55\%) predicts slip rates between 8 and $12 \mathrm{~mm} / \mathrm{yr}$, and a smaller percentage (19\%) of possible models predict fault slip rates ranging between 3 and $4 \mathrm{~mm} / \mathrm{yr}$.

After the initiation of faulting, the model allows for an acceleration in fault slip rate to occur between 14 and $0 \mathrm{Ma}$. The timing at which fault acceleration occurs is referred to as the "acceleration age" (Table 2). For the northern transect, possible acceleration ages range between 8 and $1 \mathrm{Ma}$, with a mean and mode of $6 \mathrm{Ma}$ (light gray; Figure 8a). The majority of models (77\%) show an acceleration age between 7 and 5 Ma, with the main peak centered at $6 \mathrm{Ma}$ (Figure 8a).

After the acceleration age, the post-acceleration slip rate was allowed to vary between 0 and $12 \mathrm{~mm} / \mathrm{yr}$ (Table 2). Possible postacceleration slip rates range between 1 and $11 \mathrm{~mm} / \mathrm{yr}$ with a mean of $3 \mathrm{~mm} / \mathrm{yr}$ and a mode of 2 $\mathrm{mm} / \mathrm{yr}$ (light gray; Figure 8b). The largest group of models (68\%) predicts postacceleration rates between 1 and $2 \mathrm{~mm} / \mathrm{yr}$, and a few models (12\%) predict a postacceleration rate of $3 \mathrm{~mm} / \mathrm{yr}$.

The magnitude of slip along the GMD ranges between 30 and $75 \mathrm{~km}$, with a mean between 45 and $50 \mathrm{~km}$ and a mode between 35 and $40 \mathrm{~km}$ (Figure $8 \mathrm{c}$ ). The greatest number of fits is at $30-35 \mathrm{~km} \mathrm{(32 \% ),} \mathrm{with} \mathrm{the} \mathrm{rest} \mathrm{of}$ the data forming an asymmetrical bell curve, with the peak at 50-60 km. 

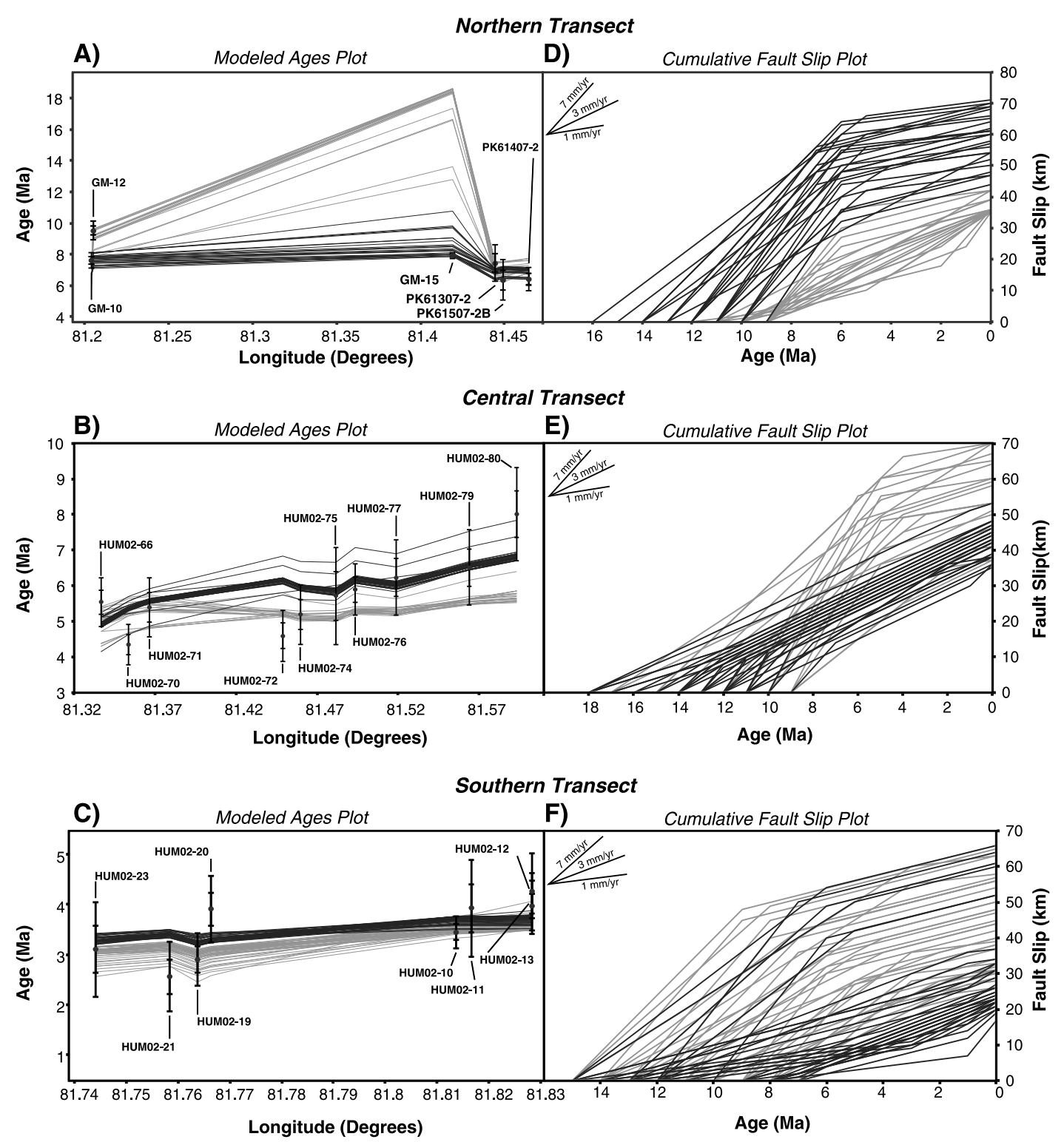

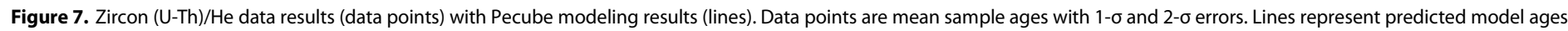

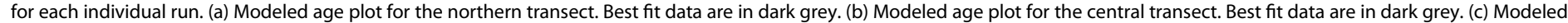

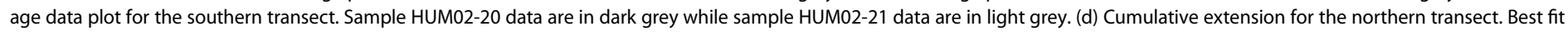

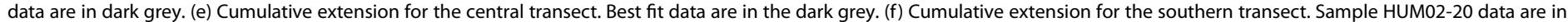
dark grey while sample HUM02-21 data are in light grey. See text for a more detailed discussion.

Figure 7a shows a group of model runs that do not match the observed age trend (light gray lines). The plot also shows a second series of model runs that reproduced the observed sample ages well, with the exception of sample GM-12. Sample GM-12 has muscovite and biotite ${ }^{40} \mathrm{Ar} /{ }^{39} \mathrm{Ar}$ ages, but the Pecube modeling was unable to reproduce the muscovite age within $2-\sigma$, and only approximately one third of the best fit models were able to reproduce the biotite age within $2-\sigma$. If we model only the data that do not drastically overestimate the biotite ${ }^{40} \mathrm{Ar} /{ }^{39} \mathrm{Ar}$ age for sample GM-15, these models reproduce the data with greater precision (dark gray, Figure 7a). Fault slip rates and initiation ages for these models show that overall trends do not change, and that the model results are more tightly constrained. These models require a fault initiation age between 12 and 11 Ma with acceleration at $6 \mathrm{Ma}$, an initial slip rate between 8 and $11 \mathrm{~mm} / \mathrm{yr}$, a postacceleration rate of 1-2 mm/yr (Figures $8 \mathrm{~d}$ and $8 \mathrm{e}$ ), and an overall net slip between 60 and $70 \mathrm{~km}$ (Figure 8f). 

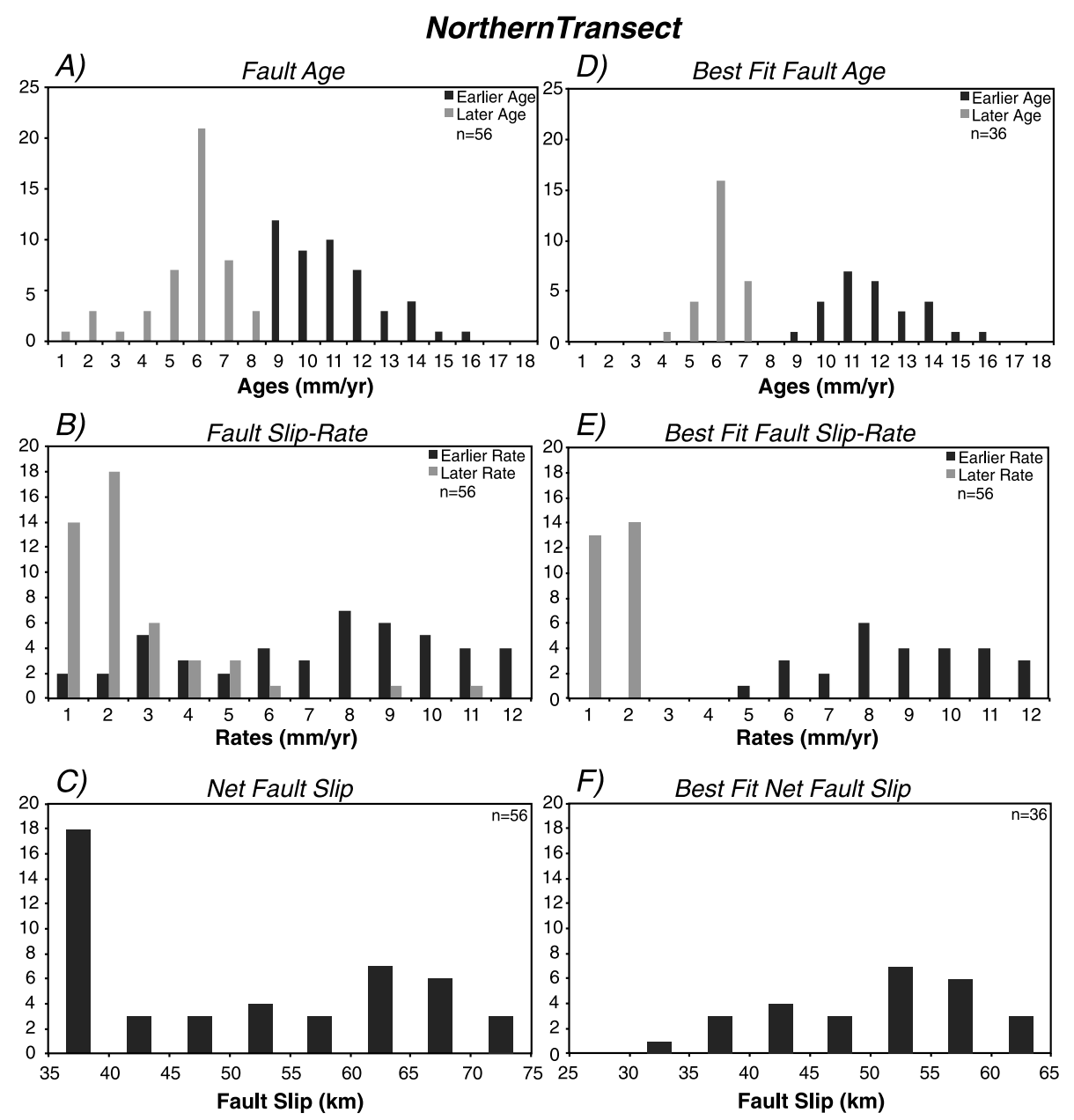

Figure 8. Pecube model results for the northern transect. Dark grey bars represent initiation parameters; light grey bars represent acceleration/postacceleration parameters. (a) Plot comparing the predicted initiation age to the acceleration age. (b) Comparison of the predicted initiation slip rate (dark grey) to the postacceleration fault slip rate (light grey). (c) Predicted net extension for the GMD. Best fit data are the model runs that do not overestimate sample GM-15, as described in section 4.2. (d) Comparison for the best fit fault initiation age to the acceleration age. (e) Comparison of the best fit fault initiation slip rate (dark grey) to the postacceleration fault slip rate (light grey). (f) Best fit net extension across the GMD. See text for a more detailed discussion.

\subsection{Central Transect}

The central transect corresponds to cross section B-B' (Figure 4b), oriented parallel to slip direction $\left(280^{\circ}\right)$, with the GMD striking $\sim 010^{\circ}$ with a $30^{\circ}$ west dip (Figure 5). A total of 12,000 models were possible. We employed the same filtering techniques described above, reducing the total number of possibilities to $\sim 6700$ model runs. (See Table 2 for model parameters.) The initial results show that 56 of 6700 models fit 8 out of 10 data points; no models were able to reproduce all 10 data points. Three samples were problematic: HUM0271, HUM02-72, and HUM02-80. Both HUM02-71 and HUM02-72 were younger than the surrounding samples, while HUM02-80 was significantly older (Figure 7b), although the ages were within 2- $\sigma$ of the surrounding samples.

The fault initiation ages range between 18 and 9 Ma with a mean of 13 Ma and a mode of 11 Ma (dark gray; Figure 9a). A majority of the best fit models (60\%) predict an initiation age between 14 and $11 \mathrm{Ma}$ (Figure 9a).

The initial-model slip rates range between 2 and $12 \mathrm{~mm} / \mathrm{yr}$, with a mean of $6 \mathrm{~mm} / \mathrm{yr}$ and a mode of $4 \mathrm{~mm} /$ yr (dark gray; Figure 9b). The largest cluster of models (50\%) predict an initial slip rate ranging between 3 and 5 $\mathrm{mm} / \mathrm{yr}$; when expanded between 2 and $7 \mathrm{~mm} / \mathrm{yr}$, the trend forms a Gaussian distribution that includes $78 \%$ of the models (Figure 9b). 

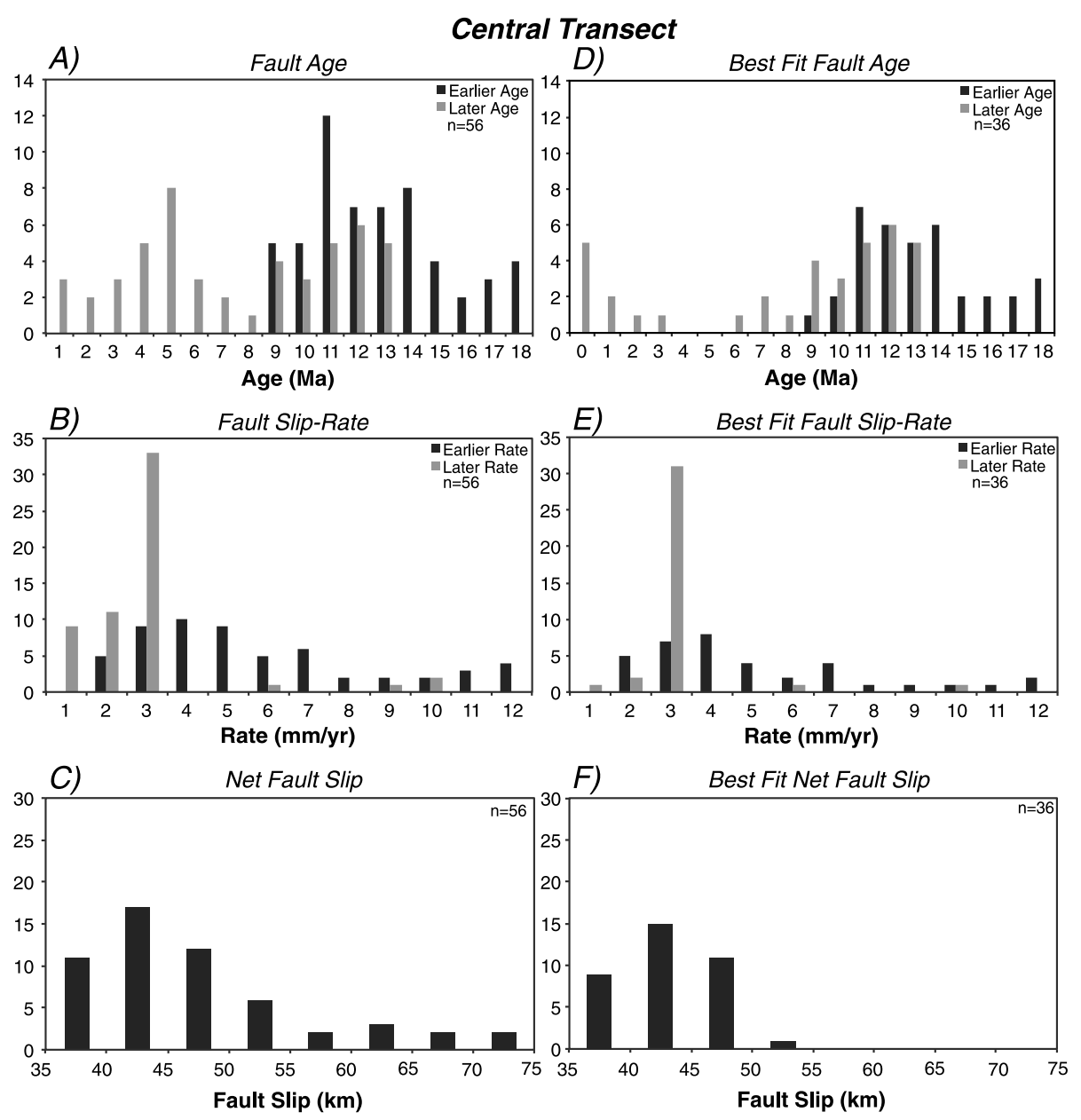

Figure 9. Pecube model results for the central transect. Dark grey bars represent fault initiation parameters, light grey bars represent acceleration and postacceleration parameters. (a) Comparison of the predicted fault initiation age to the acceleration age. (b) Comparison of the predicted initiation slip rate (dark grey) to the postacceleration slip rate (light grey). (c) Net extension across the GMD. Best fit data are the model runs that are able to capture sample HUM02-80, as described in section 4.3. (d) Comparison of the best fit fault initiation age to the acceleration age. (e) Comparison of the best fit initiation slip rate (dark grey) to the postacceleration slip rate (light grey). (f) Best fit net extension across the GMD.

The postacceleration ages range between 1 and $13 \mathrm{Ma}$, with a mean of $7 \mathrm{Ma}$ and a mode of $5 \mathrm{Ma}$ (light gray; Figure 9a). There are two main peaks: the first between 6 and $3 \mathrm{Ma}(34 \%)$ and the second between 13 and 9 Ma (41\%).

The postacceleration slip rate interval ranges between 1 and $10 \mathrm{~mm} / \mathrm{yr}$ with a mean of $3 \mathrm{~mm} / \mathrm{yr}$ and a mode of $3 \mathrm{~mm} / \mathrm{yr}$ (light gray; Figure 9b). There is a group of data ranging between 1 and $3 \mathrm{~mm} / \mathrm{yr}$ (93\%) with the largest (62\%) proportion of possible slip rates clustered at $3 \mathrm{~mm} / \mathrm{yr}$.

The model results for fault slip magnitude range between 35 and $75 \mathrm{~km}$ with a mean between 35 and $40 \mathrm{~km}$ and a mode between 40 and $41 \mathrm{~km}$ (Figure 9c). There is only one major distribution, where the majority (43\%) of models predict between 35 and $55 \mathrm{~km}$ of fault slip.

The magnitude of the fault slip shows that the models do not constrain the parameters very well. This trend is also seen in the Modeled Age (Figure 7b), but unlike the cumulative fault slip (Figure 7b), over half of the models produced a tight band (dark gray lines). Comparing the tightly grouped models, we see that the main difference is in their ability to reproduce the easternmost (last) sample in the sampling transect. We chose to weight the models based on their ability to reproduce the last sample (dark gray lines) over models that were able to reproduce the second and fourth samples (light gray lines). This is because the last sample may represent a change in the thermal history or even entrance into the partial retention zone, whereas 
the second and fourth samples deviated from their immediate surrounding samples, which renders their validity uncertain. When we remove all of the models that did not reproduce the last sample within $2-\sigma$, the precision is increased. This results in a tighter constraint on the fault initiation age between 14 and $11 \mathrm{Ma}$, with an acceleration age between 13 and $11 \mathrm{Ma}$, an initial fault slip rate between 3 and $4 \mathrm{~mm} / \mathrm{yr}$, and a postacceleration fault slip rate of $3 \mathrm{~mm} / \mathrm{yr}$ (Figures $9 \mathrm{~d}$ and $9 \mathrm{e}$ ). Within the resolution of the data, these results indicate that the fault slip rate remained constant at around $\sim 3 \mathrm{~mm} / \mathrm{yr}$, with a fault slip magnitude between 35 and $50 \mathrm{~km}$.

\subsection{Southern Transect}

The southern transect corresponds to cross section C-C' (Figure 4c). Like the northern and central transects, the model is oriented in the general slip direction $\left(280^{\circ}\right)$ with a planar fault striking $\sim 010^{\circ}$ and dipping $30^{\circ}$ west (Figure 5). Unlike in the northern and central transects, the projected updip section of the fault does not roll over after breaking the surface. This is because early model testing showed a domed fault is not required to reproduce the general age trends.

Using these initial model parameters yields a total of 12,000 possible models. Using the filtering techniques described above but increasing the possible total extension magnitude to $0-60 \mathrm{~km}$ reduces the total number of possibilities to $\sim 7700$. (See Table 2 for model parameters.) The range is decreased because field observations constraining fault slip estimates for this portion of the fault are lower (24-32 km) [Murphy and Copeland, 2005]. The initial results show that 83 of 7700 models fit 7 out of 8 data points, no models were able to reproduces all 8 data points. The two samples that the models did not reproduce well are HUM02-20 (48\%) and HUM02-21 (52\%) (Figure 7c).

Timing for fault initiation range between 15 and 7 Ma with a mean of 11 Ma and a mode of 8 Ma (dark gray; Figure 10a). The main peak of fault initiation is between 9 and $8 \mathrm{Ma}(29 \%)$, with a second wider peak spanning between 15 and $11 \mathrm{Ma}$ (48\%).

The initial fault slip rate interval allowed by the model spans from 1 to $10 \mathrm{~mm} / \mathrm{yr}$, with a mean of $4 \mathrm{~mm} / \mathrm{yr}$ and a mode of $2 \mathrm{~mm} / \mathrm{yr}$ (dark gray; Figure 10b). The largest number of possible models (52\%) requires an initial slip rate between 1 and $3 \mathrm{~mm} / \mathrm{yr}$, with a significant peak at $2 \mathrm{~mm} / \mathrm{yr}$.

For the southern transect, the timing of fault acceleration ranges between 9 and $1 \mathrm{Ma}$, with a mean of $4 \mathrm{Ma}$ and a mode of $1 \mathrm{Ma}$ (light gray; Figure 10a); 84\% of the models requires an acceleration age ranging between 6 and $1 \mathrm{Ma}$, with three major peaks at $1 \mathrm{Ma}, 3 \mathrm{Ma}$, and $6 \mathrm{Ma}$.

The postacceleration rates range between 1 and $10 \mathrm{~mm} / \mathrm{yr}$, with a mean of $3 \mathrm{~mm} / \mathrm{yr}$ and a mode of $2 \mathrm{~mm} / \mathrm{yr}$ (light gray; Figure 10b). The main group (83\%) ranges between 1 and $3 \mathrm{~mm} / \mathrm{yr}$ with a significant peak at $2 \mathrm{~mm} /$ yr.

The predicted fault slip magnitude ranges between 15 and $70 \mathrm{~km}$ with a mean of 35-40 km and a mode of 20$25 \mathrm{~km}$ (Figure 10c). The largest group (59\%) requires $20-35 \mathrm{~km}$ of fault slip. Further examination of cumulative fault slip (Figure $7 f$ ) shows that the models do not constrain slip magnitude robustly because the range varies between 10 and $60 \mathrm{~km}$. This is not the case with the Modeled Age Plot (Figure 7c), where the models are very tightly clustered, indicating a trade-off in precision between the two. The Modeled Age (Figure 7c) suggests the presence of two distinct "groups" of models. The first group is able to reproduce sample HUM02-20 $(48 \%)$ while the second is able to reproduce sample HUM02-21 (52\%) (Figure 7c). Examining the raw data (Table 1) for both data points reveals no clear reason to suspect that these zircon (U-Th)/He ages are questionable, so neither was excluded in our analysis. As with the northern and central transects, we examined each data set individually to reconcile the differences between the results of the two models.

A reexamination of cumulative fault slip (Figure 7c) shows that almost all models could reproduce sample HUM02-20 (dark gray lines) with slip magnitude ranging between 20 and $35 \mathrm{~km}$, while models reproducing sample HUM02-21 (light gray lines) predict a broader range of possible slip magnitudes (20-70 km). The models have the same results for the initiation age of faulting. This is also the case for the age of fault acceleration, with the exception of a large peak at $1 \mathrm{Ma}$ for the HUM02-20 models (Figures 10d and 10f). This trend is not observed with the initial fault slip rate, where the HUM02-20 models show a rate of 1-2 mm/yr. While the set of HUM02-21 models do not present a major peak, they predict a significantly higher rate that is $>4 \mathrm{~mm} / \mathrm{yr}$. Both sets of models agree on a postacceleration fault slip rate between 1 and $3 \mathrm{~mm} / \mathrm{yr}$. 

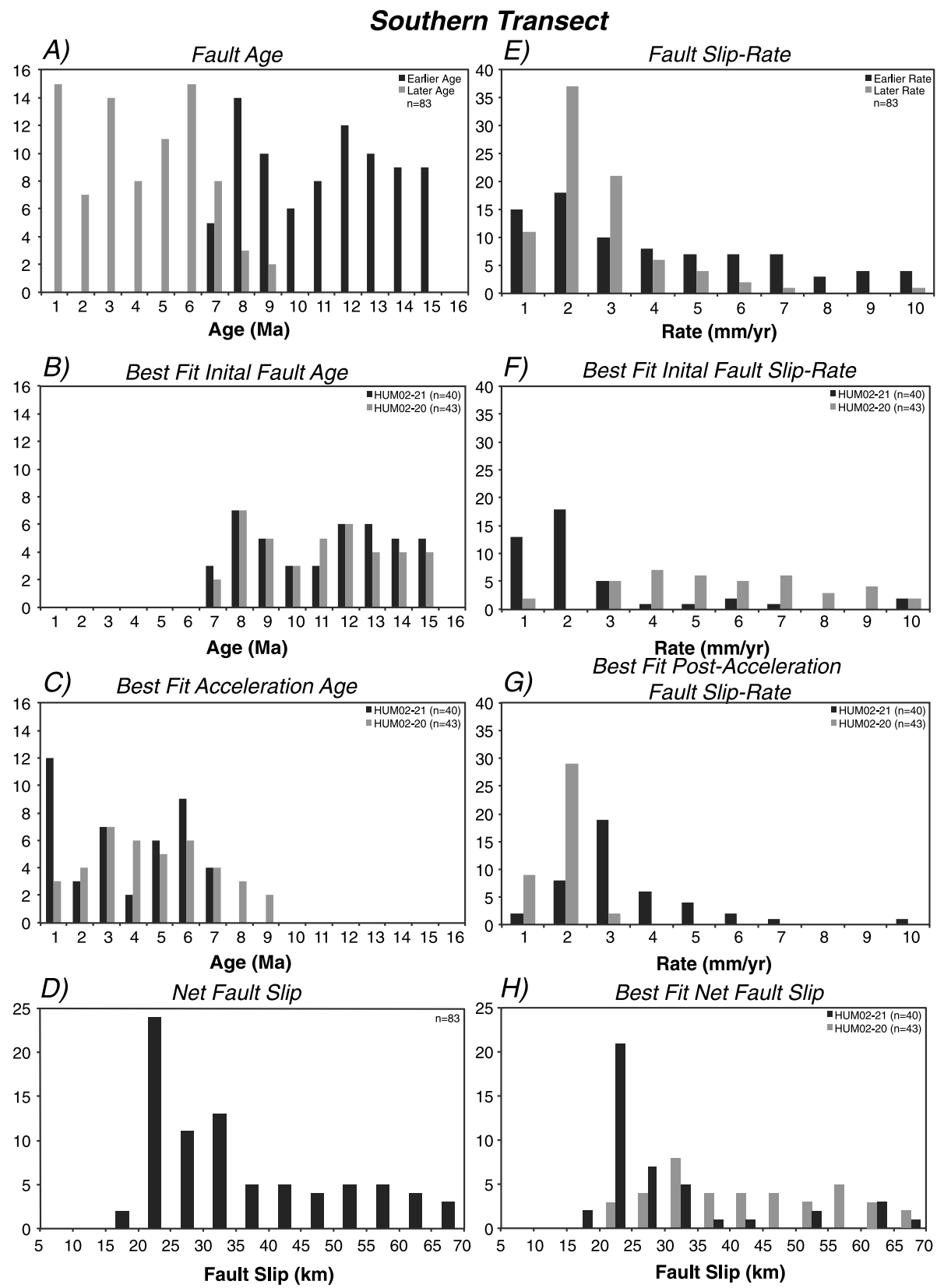

Figure 10. PeCube model results for the southern transect. (a) Comparison of the predicted initiation (dark grey) age to the acceleration age (light grey). (b) Comparison of the HUM02-20 initiation age (dark grey) to the HUM02-21 initiation age (light grey). (c) Comparison of the HUM02-20 acceleration age (dark grey) to the HUM02-21 acceleration age (light grey). (d) Predicted net extension across the GMD. (e) Comparison of the predicted initiation slip rate (dark grey) to the postacceleration slip rate (light grey). (f) Comparison of the HUM02-20 initiation slip rate (dark grey) to the HUM02-21 initiation slip rate (light grey). (g) Comparison of the HUM02-20 postacceleration slip rate (dark grey) to the HUM02-21 postacceleration slip rate (light grey). (h) Comparison of the HUM02-20 net extension (dark grey) to the HUM02-21 net extension (light grey).

The main difference between the two sets of models lies in their initial fault slip rate. Because sample HUM0220 is more definitive in its prediction of the initial fault slip rate, we give more weight to this set of models. Nonetheless, we note that we cannot exclude either model's predictions.

\subsection{Complications Within the Pecube Results}

All three Pecube models (northern, central, and southern transects) were unable to reproduce all of the sample ages. We have identified at least three potential sources of error: (1) errors within the thermochronologic 
Table 3. Table of Modeling Results and Best fit Modeling Results

\begin{tabular}{|c|c|c|c|c|c|c|}
\hline PeCube Modeling Results & Mean Fault Slip Rate & Initiation Age & Initiation Rate & Acceleration Age & Postacceleration Rate & Total Fault Slip \\
\hline Units & $\mathrm{mm} / \mathrm{yr}$ & Ma & $\mathrm{mm} / \mathrm{yr}$ & Ma & $\mathrm{mm} / \mathrm{yr}$ & $\mathrm{km}$ \\
\hline Northern Transect & $4.5 \pm 1.1$ & $14-9$ & $8-10$ & 6 & $1-3$ & 35.0 \\
\hline Central Transect & $3.8 \pm 1.2$ & $14-11$ & $3-5$ & $5-4$ & 3 & 40.0 \\
\hline Southern Transect & $3.2 \pm 1.3$ & $15-8$ & $1-3$ & $7-4$ & $1-3$ & $20-35$ \\
\hline Northern Transect Best Fit & $5.0 \pm 0.9$ & $14-11$ & $8-11$ & 6 & $1-2$ & $58-69$ \\
\hline Central Transect Best Fit & $3.3 \pm 0.6$ & $14-11$ & $2-4$ & $13-11$ & 3 & $40-46$ \\
\hline Southern Transect Best Fit & $2.7 \pm 1.1 / 3.8 \pm 1.0$ & $15-8$ & $1-2$ & $6-3$ & $2-4$ & $20-35 / 25-65$ \\
\hline
\end{tabular}

ages, (2) complications within the Pecube models themselves, and (3) an over simplification of the fault system and inherited assumptions in fault geometry. The first potential reason for this inaccuracy is within the thermochronologic data themselves. The model zircon (U-Th)/He thermochronometric age could differ from the "actual age" due to enrichment in parent isotopes of the zircon's rim. This enriched rim, possibly sourced from partial melts within the Himalaya, would result in the measured age being younger than the "actual age" [Reiners, 2005]. Thus, any samples measured from the intrusive leucogranites or grains with a complex growth history could produce ages that are incorrect.

The second source of error could result from the following complications within the Pecube modeling approach. (1) Because most of the samples were collected on or near the valley floor, the smoothed DEM that we import into the model could have misplaced the "modeled" sample location 10's-100's of meters above the actual sample location. (2) The modeling also assumes all slip along the modeled fault is pure normal slip. (3) The geometry of the fault plane is assumed to be planar. These factors could result in the model's inability to reproduce all of the sample ages.

The final source is the assumptions and over simplification of the fault system. The main assumption within this modeling is that all of the exhumation is driven by the GMD. But, other processes could influence the exhumation history of the footwall samples: (1) isostatic rebound due to erosion of topography, (2) corrugations within the footwall, and (3) exhumation related to slip along older shortening structures (e.g., the Great Counter Thrust, the Main Central Thrust, and the orogen-parallel South Tibetan detachment system).

\section{Discussion}

\subsection{Development of the Gurla Mandhata Detachment}

The zircon (U-Th)/He data collected from the GMD footwall samples show that the footwall rocks cooled below $175-190^{\circ} \mathrm{C}$ by Late Miocene to Pliocene time and are consistent with rapid cooling of the entire footwall. Possible ways to account for this age trend include the following: (1) allow for extremely fast exhumation of the footwall and (2) compress the lower temperature isotherms toward the surface. It is likely that both of these processes occurred during the structural development of the GMD. Based on the high $U$ and Th concentration in the zircons processed (Table 1) in this study as well as the radiogenic heating measurements of Himalayan rocks [Faccenda et al., 2008], it is very likely that the crust below Gurla Mandhata has a higher than average geothermal gradient. When this factor is combined with the predicted slip rate for the GMD, we would expect "hot" footwall rocks to be exhumed to the surface at a fast enough rate to cause compression of the isotherms in the GMD footwall.

Our thermal modeling indicates fault initiation occurred between 14 and $11 \mathrm{Ma}$ (Table 3), with a general trend of decreasing initiation age from north to south, and maximum net slip in the north between 58 and $69 \mathrm{~km}$ (Figure 7 and Table 3). Our estimates for initiation of faulting and maximum slip along the GMD system are consistent with previous work [Murphy et al., 2002]. The models predict initial slip rates from 8.0 to $11.0 \mathrm{~mm} / \mathrm{yr}$ for the northern transect, and these rates decrease to 1-2 mm/yr since $\sim 6 \mathrm{Ma}$ ago. In the central transect, the models predict a continuous slip rate between 3 and $4 \mathrm{~mm} / \mathrm{yr}$; in the southern transect, the models predict that the fault has a similar slip rate of $1-3 \mathrm{~mm} / \mathrm{yr}$ throughout the duration of exhumation. The initial rates for the northern and central transects bear similarity to the long-term geologic slip rates reported by Murphy et al. [2002], while the mean slip rates and postacceleration rates for all three transects are similar to the Quaternary slip rates reported by Chevalier et al. [2012] (Table 4). 
Table 4. Table of Gurla Mandhata (GMD) and Karakoram Fault (KF) Slip Rates Corresponding to Figure 11

\begin{tabular}{|c|c|c|c|c|c|c|}
\hline Fault & Location & Timing & Methods & Rates $(\mathbf{m m} / \mathbf{y r})$ & Source & Symbol \\
\hline GMD & Northern Transect & $12-6 / 6-0 \mathrm{Ma}$ & (U-Th)/He & $5.9-4.1$ & This study & NT \\
\hline GMD & Central Transect & $14-0 \mathrm{Ma}$ & (U-Th)/He & $3.9-2.7$ & This study & $\mathrm{CT}$ \\
\hline GMD & Southern Transect & $11-0 \mathrm{Ma}$ & (U/Th)/He & $1.6-4.8$ & This study & ST \\
\hline KF & Northern & $23-0 \mathrm{Ma}$ & & $6-12$ & Robinson [2009] & R9 \\
\hline KF & Northern & $15-0 \mathrm{Ma}$ & $\mathrm{U}-\mathrm{Pb}$ & $2.7-10.2$ & [Phillips et al. [2004]] & P4 \\
\hline KF & Northern & $15-0 \mathrm{Ma}$ & $\mathrm{U}-\mathrm{Pb}$ & $3-10$ & Rutter et al. [2007] & R7 \\
\hline KF & Central & $23-0 \mathrm{Ma}$ & ${ }^{40} \mathrm{Ar} /{ }^{39} \mathrm{Ar}, \mathrm{FT}, \mathrm{U}-\mathrm{Pb}$ & $7-13$ & Lacassin et al. [2004] & L4 \\
\hline KF & Central & $23-0 \mathrm{Ma}$ & ${ }^{40} \mathrm{Ar} /{ }^{39} \mathrm{Ar}, \mathrm{FT}, \mathrm{U}-\mathrm{Pb}$ & $7-10$ & Valli et al. [2007, 2008] & V7 \\
\hline KF & Central & $18-0 \mathrm{Ma}$ & ${ }^{40} \mathrm{Ar} /{ }^{39} \mathrm{Ar}, \mathrm{U}-\mathrm{Pb}$ & $7.3-9.3$ & Searle et al. [1998] & S98 \\
\hline $\mathrm{KF}$ & Central & $13-0 \mathrm{Ma}$ & ${ }^{40} \mathrm{Ar} /{ }^{39} \mathrm{Ar}, \mathrm{U}-\mathrm{Pb}$ & $7-15$ & Wang et al. [2011] & W11 \\
\hline KF & Central & 200 ka & ${ }^{10}$ Be Cosmogenics & $6-11$ & Chevalier et al. [2005a, 2005b] & $\mathrm{CH} 5$ \\
\hline KF & Central & $12 \mathrm{ka}$ & ${ }^{10}$ Be Cosmogenics & $8-10$ & Chevalier et al. [2011] & $\mathrm{CH} 11$ \\
\hline KF & Central & $14 \mathrm{ka}$ & ${ }^{10} \mathrm{Be}$ Cosmogenics & $3-5$ & Brown et al. [2002] & B2 \\
\hline KF & Central & $<10 \mathrm{a}$ & GPS & $7-15$ & Banerjee and Burgmann [2002] & BB2 \\
\hline KF & Central & $<10 \mathrm{a}$ & GPS & $3-5$ & Chen et al. [2004] & $\mathrm{C} 4$ \\
\hline KF & Central & $<10 \mathrm{a}$ & GPS & $0-8$ & Jade et al. [2004, 2010] & $\mathrm{J} 10$ \\
\hline KF & Central & $<10 \mathrm{a}$ & GPS & $3-5.5$ & Loveless and Meade [2011] & L11 \\
\hline KF & Central & $<10 a$ & InSAR & $1-4$ & Wright et al. [2004] & W4 \\
\hline KF & Central & $<10 \mathrm{a}$ & InSAR & $0-8$ & Wang and Wright, 2012 & WW12 \\
\hline KF & Central & $<10$ a & GPS & $4-10$ & Zhang et al. [2004] & Z4 \\
\hline $\mathrm{KF}$ & Southern & $13-0 \mathrm{Ma}$ & ${ }^{40} \mathrm{Ar} /{ }^{39} \mathrm{Ar}$ & $4.0-6.0$ & Murphy et al. [2000] & MO \\
\hline $\mathrm{KF}$ & Southern & $12-0 \mathrm{Ma}$ & ${ }^{40} \mathrm{Ar} /{ }^{39} \mathrm{Ar}, \mathrm{U}-\mathrm{Pb}$ & $5-9$ & Wang et al. [2009] & W9 \\
\hline KF & Southern & $12-0 \mathrm{Ma}$ & $\mathrm{U}-\mathrm{Pb}$ & $4.4-4.6$ & Wang et al. [2012] & W12 \\
\hline KF & Southern & $<100$ ka & ${ }^{10}$ Be Cosmogenics & $5-11$ & Chevalier et al. [2012] & $\mathrm{CH} 12 \mathrm{a}$ \\
\hline GMD & Southern & $15-0 \mathrm{Ma}$ & ${ }^{40} \mathrm{Ar} /{ }^{39} \mathrm{Ar}$ & $1.4-4.6$ & Murphy and Copeland [2005] & MC5 \\
\hline GMD & Northern / Central & $13-0 \mathrm{Ma}$ & ${ }^{40} \mathrm{Ar} /{ }^{39} \mathrm{Ar}$ & $2.7-7.3$ & Murphy et al. [2002] & M2 \\
\hline GMD & Northern / Central & $40 \mathrm{ka}$ & ${ }^{10}$ Be Cosmogenics & ${ }^{\mathrm{a}} 4.0-2.6$ & Chevalier et al. [2012] & $\mathrm{CH} 12 \mathrm{~b}$ \\
\hline
\end{tabular}

${ }^{\mathrm{a}}$ Slip rate calculated assuming a fault dip of 30 .

The modeling results from the northern, central, and southern transects are consistent with initiation of the GMD beginning in the north, followed by southward propagation of the detachment system over time. This trend is evident upon examination of the best fit data (dark grey lines) in the Cumulative Extension plots (Figure 7), which reveal that net fault slip drops from 58-69 km in the north, to $17-35 \mathrm{~km}$ in the south. The highest mean slip rates of the three transects revealed in the northern transect are $5.0 \pm 0.9 \mathrm{~mm} / \mathrm{yr}$ and appear to decrease to $3.2 \pm 1.6 \mathrm{~mm} / \mathrm{yr}$ for the southern transect. Collectively, the north to south gradient in slip magnitude and slip rate suggests that the GMD initiated in the north and propagated southward.

We note that the combination of thermal parameters produces a pre-extensional temperature with a geothermal gradient of $20-25^{\circ} \mathrm{C} / \mathrm{km}$ in the upper $20 \mathrm{~km}$ of the crust, which is typical for continental crust. These values are somewhat lower than what has been used in Pecube models in other locations in the southwestern Himalayan-Tibetan orogen [e.g., Styron et al., 2013; Herman et al., 2010]. However, following extension on the Gurla Mandhata detachment, the thermal field approaches that used in other studies [e.g., Styron et al., 2013; Herman et al., 2010]. A hotter geotherm in our model would result in lower slip rates to reproduce the observed cooling ages, but our results are consistent with low slip rates compared with other studies suggesting a moderate geothermal gradient as opposed to a hotter one $\left(\sim 40-50^{\circ} \mathrm{C} / \mathrm{km}\right)$ [e.g., Styron et al., 2013; Herman et al., 2010]. Therefore, even though individual thermal parameters are not as constrained as we would like, the resultant geotherm is quite reasonable.

\subsection{Comparison with other Himalayan Extensional Structures}

The modeled initiation age for the GMD is within

$\pm 2 \mathrm{Ma}$ of that estimated for the Ama Drime detachment system [Jessup et al., 2008; Langille, et al., 2010]. In addition, the Pliocene exhumation rate for the GMD is within $\pm 1 \mathrm{~mm} / \mathrm{yr}$ of that estimated for Ama Drime [Jessup et al., 2008; Langille et al., 2010]. Both Ama Drime and the GMD have 10s of km of displacement associated with the main fault that results in the exhumation of midcrustal Himalayan rocks [Murphy et al., 2002, Murphy and Copeland, 2005; Murphy, 2007; Jessup et al., 2008; Langille et al., 2010]. Both extensional systems have similar ages obtained from biotite and muscovite ${ }^{40} \mathrm{Ar} /{ }^{39} \mathrm{Ar}$ data [Murphy et al., 2002; Jessup et al., 2008]. Although the GMD and Ama Drime extensional systems are significantly separated along the Himalayan arc, they share very similar histories and structural characteristics. 
To the west of the GMD is the Leo Pargil dome (Figure 1). ${ }^{40} \mathrm{Ar} /{ }^{39} \mathrm{Ar}$ muscovite and biotite ages from the footwall of the Leo Pargil dome range between $14.5 \pm 0.1$ and $15.5 \pm 0.1 \mathrm{Ma}$, and apatite FT ages range between $1.7 \pm 0.3$ and $9.9 \pm 0.8 \mathrm{Ma}$ [Thiede et al., 2006]. The ${ }^{40} \mathrm{Ar} /{ }^{39} \mathrm{Ar}$ sample ages are much older than that observed for the GMD, and while the FT ages span a larger range, the results are consistent with the lower-temperature (U/ Th)/He ages obtained from the GMD footwall. This range in ages has been interpreted to represent a threestage development beginning with rapid cooling between 16 and $14 \mathrm{Ma}$, followed by slow exhumation rates (0.07-0.16 mm/yr) between 10 and $4 \mathrm{Ma}$, and finally with rapid exhumation $(0.4-1.9 \mathrm{~mm} / \mathrm{yr})$ from 4 to $0 \mathrm{Ma}$ [Thiede et al., 2006]. Recent work has suggested that exhumation of the footwall could have started as early as $23 \mathrm{Ma}$ [Langille et al., 2012]. These exhumation rates and younger timing relationships of the Leo Pargil dome are comparable to slower predicted fault slip rates from the Pecube modeling of the GMD. From these results, it seems that the GMD and the Leo Pargil dome have slightly different faulting histories, with the Leo Pargil fault initiating earlier and slipping at slower rates.

The modeled GMD slip rates are comparable to slower slip rate estimates for the Kung Co fault system (Figure 1). Thermochronologic data from the footwall of the Kung Co fault show zircon (U-Th)/He ages ranging between 12.9 and 8.3 Ma and Apatite (U-Th)/He ages ranging between 17.5 and 3.1 Ma [Lee et al., 2011]. The Kung Co fault initiated approximately $13 \mathrm{Ma}$ ago had a period of high fault slip rates ( $>7 \mathrm{~mm} / \mathrm{yr}$ ) between 13 and $10 \mathrm{Ma}$, and then slowed from 9 to $0 \mathrm{Ma}$ [Lee et al., 2011]. Both fault systems yield similar ages for faulting initiation, but only the lowest rates modeled on the Kung Co fault are comparable to the modeling results for the GMD system.

\subsection{Comparison of GMD to KF}

For our purposes, the slip-rate data from the southern portion of the KF are most relevant to this study. Slip rates inferred from geodesy for the central KF have mixed results with lower bounds ranging between 1 and 6 $\mathrm{mm} / \mathrm{yr}$ [Chen et al., 2004; Wright et al., 2004; Loveless and Meade, 2011; Wang and Wright, 2012], to upper bounds between 5 and 15 mm/yr [Banerjee and Burgmann, 2002; Jade et al., 2004; Zhang et al., 2004]. Fault slip rate estimates based on ${ }^{10} \mathrm{Be}$ cosmogenic nuclide dating of boulders from offset geomorphic landforms have also produced both low [Brown et al., 2002] and high fault slip rates [Chevalier et al., 2005a, 2005b, 2011, 2012], which are strongly dependent on the preferred offset reconstructions and sampling strategy [e.g., Brown, 2005]. The long-term geologic slip rates for the southern KF range between 4.7 and $9 \mathrm{~mm} / \mathrm{yr}$ [Murphy et al., 2000; Wang et al., 2009, 2012]; for the central KF, these values range between 7 and $15 \mathrm{~mm} / \mathrm{yr}$ [Searle et al., 1998; Lacassin et al., 2004; Valli et al., 2007, 2008; Wang et al., 2011].

The mean modeled slip rates in this study match slip rate estimates for the southern $\mathrm{KF}$ across different timescales (Figure 11). Some of the best rate agreements are with the geodetic loading rates [Chen et al., 2004; Wright et al., 2004; Zhang et al., 2004; Jade et al., 2004, 2010; Loveless and Meade, 2011] and the slower, long-term fault slip rates [Murphy et al., 2000; Phillips et al., 2004; Rutter et al., 2007; Wang et al., 2012], while only a single slip rate using cosmogenic nuclides is in agreement with our mean modeled fault slip rate (Figure 11) [Brown et al., 2002]. The individual transect results show that the fault slip rates obtained for the northern transect match all of the KF slip rate data, but this is mostly due to the very high fault slip rate associated with initiation of the GMD. Both the central and southern transects have slower fault slip rates $(<5$ $\mathrm{mm} / \mathrm{yr}$ ) that are in agreement with shorter timescales obtained with cosmogenic nuclides and geodesy [Chevalier et al., 2012] (Figure 11). We note that numerous elastic block models have been constructed to model GPS velocities across the significant faults of the Tibetan-Himalayan orogeny to obtain long-term fault slip rates [e.g., Loveless and Meade 2011; Gan et al., 2007]. In the elastic block models, southwest Tibet is simplified by omitting the GMD fault, and fault slip is transferred into and along the Indus-Yalu suture zone, which we discuss in more detail in section 5.5.1.

From these results, there is compelling evidence that the GMD (14-11 Ma) and the southern portion of the KF [ 13 Ma; Murphy et al., 2000] have similar ages of fault initiation and magnitudes of fault slip. In addition, the fault slip rates modeled for the GMD are comparable to the slip rate data for the central and southern KF across several timescales (10-106 years) (Figure 11). These results suggest that the GMD has been kinematically linked to the KF since 14-11 Ma and that the GMD transfers slip southeastward into the Humla fault and High Himalaya. 


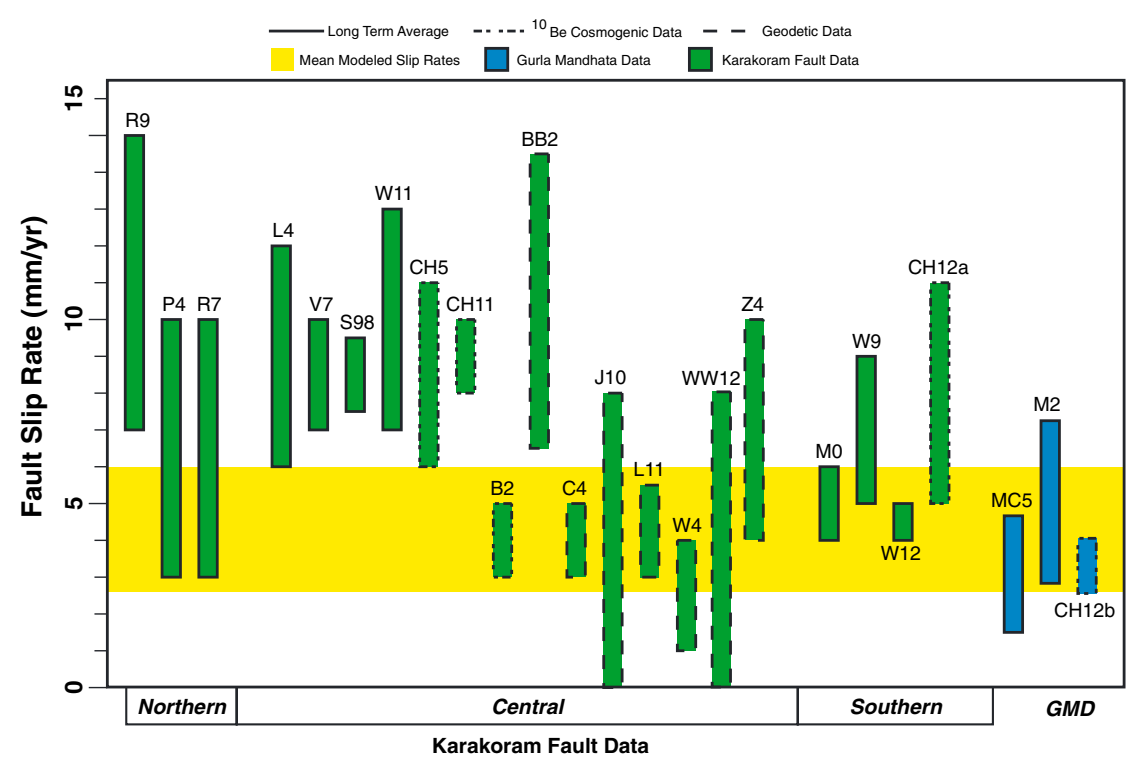

Figure 11. Comparison of fault slip-rate estimates for the KF and GMD. Blue bars denote data for the GMD, green bars are data for the KF, and yellow bar represents the mean slip rates of the three models. Data representing the long-term slip rate have a solid border, cosmogenic data have a stippled border, and GPS data have a dashed border. The data are organized based on their geographic location for each fault. The Karakoram Fault data are organized into three groups: northern data, central data, and southern data. Within each group, the data are organized from $10^{6}$ to $10^{1}$ time scales. The references for the data can be found in Table 4. B2 - Brown et al. [2002]; BB2 - Banerjee and Burgmann [2002]; C4 Chen et al. [2004]; CH5 - Chevalier et al. [2005a, 2005b]; CH11 - Chevalier et al. [2011]; CH12a - Chevalier et al. [2012]; CH12b - Chevalier et al. [2012]; J10 - Jade et al. [2004, 2010]; L4 - Lacassin et al. [2004]; L11 - Loveless and Meade [2011]; M0 - Murphy et al. [2000]; M2 - Murphy et al. [2002]; MC5 - Murphy and Copeland [2005]; P4 - Phillips et al. [2004]; R7 - Rutter et al. [2007]; R9 - Robinson [2009]; S98 - Searle et al. [1998]; V7 - Valli et al. [2007, 2008]; W4 Wright et al. [2004]; W9 - Wang et al. [2009];W11 - Wang et al. [2011]; W12 - Wang et al. [2012]; WW12 - Wang and Wright [2012]; Z4 - Zhang et al. [2004].

\subsection{Implications for Tectonic Models}

To thoroughly evaluate the viability of the tectonic models described in section 1.1, we compare the results from this study to the predictions made by each of the four models beginning with lateral extrusion, oroclinal bending, radial spreading, and finally oblique convergence. Collectively, understanding the role of the GMD system in the above mentioned models gleans further insight into the significance of active extensional structures in the hinterlands of orogenic systems.

\subsubsection{Lateral Extrusion}

The lateral extrusion model predicts high rates $(>1 \mathrm{~cm} / \mathrm{yr}$ ) and magnitudes (100's of km) of dextral slip on both the KF and IYS zone. The model also describes that most of the dextral motion associated with the KF is transferred into the IYS zone. The results of this study show that the GMD accommodates the entire dextral shear associated with the southern KF, which implies that negligible strain is transferred along the IYS past the GMD since the mid Miocene. Additionally, there is strong doubt that the IYS is an active dextral structure based on the lack of any compelling evidence for active faulting along the IYS east of longitude $82.3^{\circ}$ (Figure 12), and clear crosscutting field relationships documented at the southern end of the Lopukangri rift, where the IYS zone and Great Counter thrust are cut and offset by the N-striking Lopukangri normal fault [Murphy et al., 2010].

\subsubsection{Oroclinal Bending}

Comparing our results with those predicted by the oroclinal bending model, we see that our results do not match its predictions. This model predicts that extension rates should increase toward the frontal portions of the thrust belt. However, when our data are combined with geodetic data for western Nepal [Larson et al., 1999; Jouanne et al., 2004], there is an apparent decrease in dextral shear toward the Himalayan front. Also, the predicted kinematics for the KF, faults in central Tibet, and southeastern Tibet do not match field observations. In particular, model predictions require sinistral motion on the KF, which are opposite to field observations of dextral faulting [Ratschbacher et al., 1994; Searle et al., 1998; Murphy et al., 2000]. 

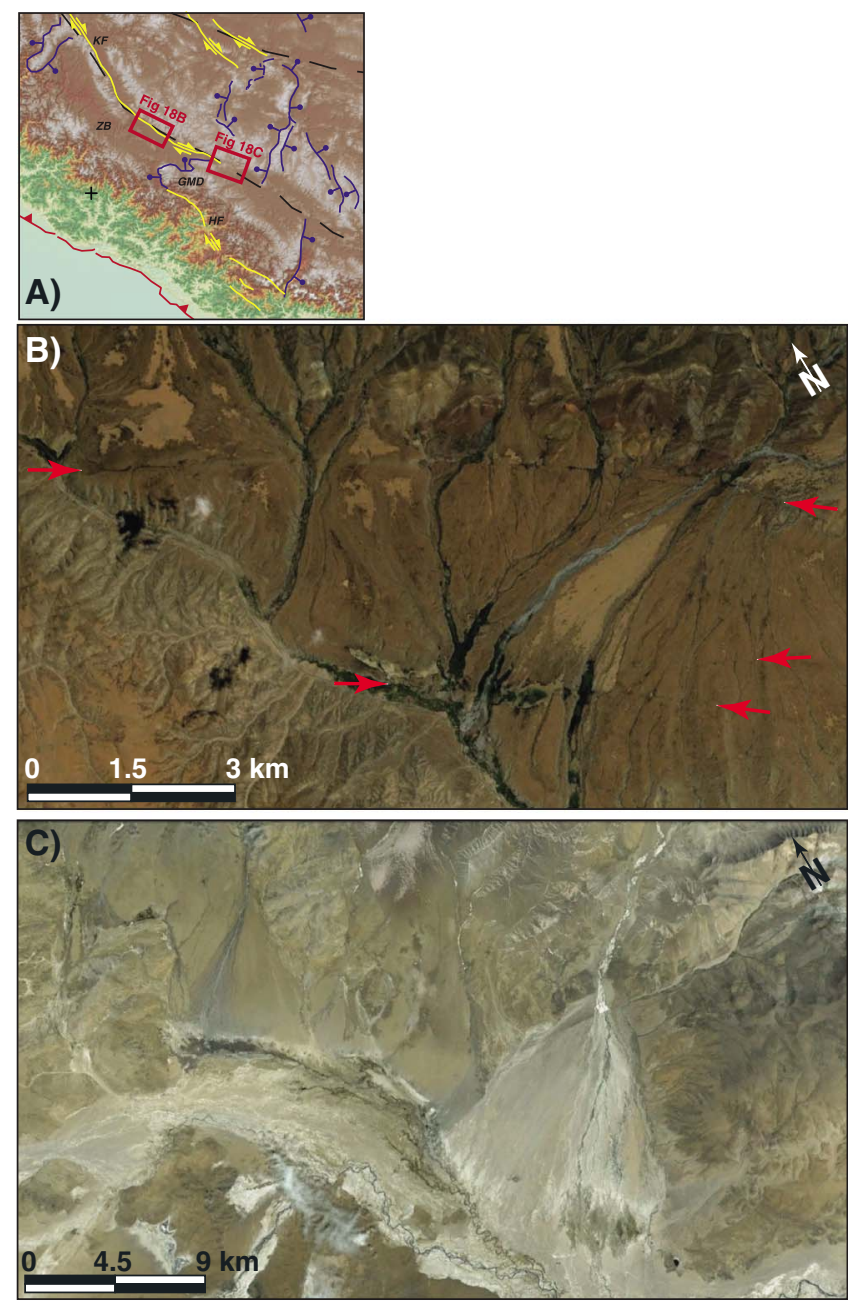

Figure 12. (a) Shaded relief map of the Tibetan plateau showing the major fault distributions. Thrust faults are in red, normal faults in blue, and strike-slip faults in yellow. The dashed black lines represent the suture zones. The red boxes mark the locations for Figure 12b and 15c. GMD - Gurla Mandhata Detachment; HF Humla fault; KF - Karakoram fault; ZB - Zada Basin. Modified after Taylor et al. [2003] and Taylor and Yin [2009]; faults taken from Styron et al. [2010]. (b) Image of the Karakoram fault north of the Kailas Thrust. Note the offset river terraces and fluvial channels. Image taken from Google Earth. (c) Satellite image northeast of the GMD where the IYS is interpreted to be. Note the lack of active fault traces and there are no apparent offsets of the alluvial fans and active river systems. Image taken from Google Earth.

in the arc-parallel component of displacement west of the central Himalaya. Recent GPS compilations and analysis [Gan et al., 2007, Styron et al., 2011] indicate an increase is the arc-parallel component of the surface displacement field west of the central Himalaya. Our study indicates that the GMD and KF systems are acting together as a kinematically coordinated fault system, which is also consistent with oblique convergence. While beyond the scope of this study, our finding is important for understanding the processes and rates associated with arc-parallel extension in the hinterlands of orogenic systems, which is a more important process than previously thought.

\section{Conclusions}

Our study in southwest Tibet along the GMD is summarized by the following points:

1. (U-Th)/He dating of zircon from 21 samples collected along three transects yields ages between $8.0 \pm 1.3$ and $2.6 \pm 0.7 \mathrm{Ma}$, consistent with rapid exhumation of the GMD footwall. The sample transects 
were evaluated using the Pecube finite element software, which was used to run $7700-6700$ models per transect to constrain the initiation and slip history for the GMD. Our results are consistent with a southward propagating history, with the highest net slip $(50-60 \mathrm{~km})$ in the north that decreases to less than half that at its southernmost segment (15-30 km). Modeling results for fault slip rates for the central and southern segments are similar with slower fault slip rates $(\sim 1-4 \mathrm{~mm} / \mathrm{yr})$, while the northern segment experiences higher rates of faulting $(5.0 \pm 0.9 \mathrm{~mm} / \mathrm{yr})$.

2. Gurla Mandhata is one of several examples for demonstrating how arc parallel extension is accommodated in the High Himalaya. Examples of other extensional structures along the High Himalaya near the Tibetan border include the Leo Pargil and Ama Drime domes, and the Kung Co rift, all of which initiated between 16 and $9 \mathrm{Ma}$. While the above mentioned structures do not have a direct linkage with the Karakoram fault system, this and previous studies demonstrate that the structural and kinematic history of the GMD fault system is comparable to the other Himalayan extensional structures thus highlighting the importance of active extension within the Himalayan thrust wedge.

3. The kinematic relationships obtained from Pecube modeling results indicate that the GMD system had a similar initiation age and slip rate compared with the Karakoram fault, consistent with the GMD acting as a right-step extensional feature within a southward propagating system of dextral shear. If this relationship between the GMD and Karakoram fault is correct, this structural relationship requires that fault slip bypasses the IYS zone and transfers slip into the High Himalaya. Furthermore, this model is most consistent with active faults in southwest Tibet and western Nepal acting in the context of oblique convergence.

Acknowledgments

The authors appreciate comments from two anonymous reviewers and Editor Todd Ehlers who helped to improve the overall presentation of this study. Numerous discussions with Andreas Moeller, J.D. Walker, Paul Kapp, Alexander Robinson, and Alexander Pullen helped tremendously in shaping our thoughts. This work was supported by the Tectonics Division of the National Science Foundation (EAR0809408 and EAR-0911652) to M. Taylor.

\section{References}

Banerjee, P., and R. Burgmann (2002), Convergence across the northwest Himalaya from GPS measurements, Geophys. Res. Lett., 29(13), 1652, doi:10.1029/2002GL015184.

Besse, J., V. Courtillot, J. P. Pozzi, M. Westphal, and Y. X. Zhou (1984), Palaeomagnetic estimates of crustal shortening in the Himalayan thrusts and Zangbo suture, Nature, 311, 621-626.

Braun, J., et al. (2012), Quantifying rates of landscape evolution and tectonic processes by thermochronology and numerical modeling of crustal heat transport using PECUBE, Tectonophysics, 524, 1-28.

Braun, L. (2003), Pecube: a new finite-element code to solve the 3D heat transport equation including the effects of a time-varying, finite amplitude surface topography, C. R. Geosci., 29, 787-794.

Brown, E. T. (2005), Comment on "Slip-Rate Measurements on the Karakorum Fault May Imply Secular Variations in Fault Motion", Science, 309, 1326, doi:10.1126/science.1112508.

Brown, E. T., R. Bendick, D. L. Bourles, V. Gaur, P. Molnar, G. M. Raisbeck, and F. Yiou (2002), Slip rates of the Karakorum fault, Ladakh, India determined using cosmic ray exposure dating of debris flows and moraines, J. Geophys. Res., 107(B9), 2192, doi:10.1029/2000JB000100.

Chen, Q., J. T. Freymueller, Z. Yang, C. Xu, W. Jiang, Q. Wang, and J. Liu (2004), Spatially variable extension in southern Tibet based on GPS measurements, J. Geophys. Res., 109, B09401, doi:10.1029/2002JB002350.

Chen, W. P., M. Martin, T. L. Tseng, R. L. Nowack, S. H. Hung, and B. S. Huang (2010), Shear-wave birefringence and current configuration of converging lithosphere under Tibet, Earth Planet. Sci. Lett., 295, 297-304.

Chevalier, M. L., F. J. Ryerson, P. Tapponnier, R. C. Finkel, J. Van Der Woerd, L. Haibing, and L. Qing (2005a), Slip rate measurements on the Karakorum fault may imply secular variations in fault motion, Science, 307, 411-414.

Chevalier, M.-L., F. J. Ryerson, P. Tapponnier, R. C. Finkel, J. Van Der Woerd, L. Haibing, and L. Qing (2005b), Response to comment on "Slip rate measurements on the Karakorum fault may imply secular variations in fault motion", Science, 309, 1326, doi:10.1126/ science. 1112629.

Chevalier, M.-L., H. Li, J. Pan, J. Pei, F. Wu, W. Xu, Z. Sun, and D. Liu (2011), Fast slip rate along the northern end of the Karakorum fault system, western Tibet, Geophys. Res. Lett., 38, L22309, doi:10.1029/2011GL049921.

Chevalier, M. L., P. Tapponnier, J. V. Woerd, F. Ryerson, R. C. Finkel, and H. Li (2012), Spatially constant slip rate along the southern segment of the Karakorum fault since 200 ka, Tectonophysics, 530-531, 152-179.

Faccenda, M., T. V. Gerya, and S. Shakraborty (2008), Styles of post-subduction collisional orogeny: Influence of convergence velocity, crustal rheology and radiogenic heat production, Lithos, 103, 257-287.

Gaetani, M., and E. Garzanti (1991), Multicyclic History of the Northern India Continental Margin [Northwestern Himalaya], Am. Assoc. Pet. Geol. Bull., 75, 1427-1446.

Gan, W., P. Zhang, Z. Shen, Z. Niu, M. Wang, Y. Wan, D. Zhou, and J. Cheng (2007), Present-day crustal motion within the Tibetan Plateau inferred from GPS measurements, J. Geophys. Res., 112, B08416, doi:10.1029/2005JB004120.

Herman, F., et al. (2010), Exhumation, crustal deformation, and thermal structure of the Nepal Himalaya derived from the inversion of thermochronological and thermobarometric data and modeling of topography, J. Geophys. Res., 115, B06407, doi:10.1029/2008JB006126.

Jade, S., B. C. Bhatt, Z. Yang, R. Bendick, V. K. Gaur, P. Molnar, B. M. Anand, and D. Kumar (2004), GPS measurements from the Ladakh Himalaya India: Preliminary test of plate-like or continuous deformation in Tibet, Geol. Soc. Am. Bull., 116, 1385-1391.

Jade, S., R. H. J. Raghavendra Rao, M. S. M. Vijayan, V. K. Gaur, B. C. Bhatt, K. Kumar, S. Jaganathan, M. B. Ananda, and P. Dileep Kumar (2010), GPS-derived deformation rates in northwestern Himalaya and Ladakh, Int. J. Earth Sci., 100, 1293-1301, doi:10.1007/s00531-010-0532-3.

Jessup, M. J., and J. M. Cottle (2010), Progression from South-Directed Extrusion to Orogen-Parllel Extension in the Southern Margin of the Tibetan Plateau, Mount Everest Region, Tibet, J. Geol., 118, 467-486, doi:10.1086/655011.

Jessup, M. J., D. L. Newell, J. M. Cottle, A. L. Berger, and J. A. Spotila (2008), Orogen-parallel extension and exhumation enhanced by denudation in the trans-Himalayan Arun River gorge, Ama Drime Massif, Tibet-Nepal, Geology, 36, 587-590.

Jouanne, F., J. L. Mignier, J. F. Gamond, P. Le Fort, M. R. Pandey, L. Bollinger, M. Flouzat, and J. P. Avouac (2004), Current Shortening across the Himalayas of Nepal, Geophys. J. Int., 157, 1-14. 
Klootwijk, C. T., P. J. Conaghan, and C. M. Powell (1985), The Himalayan Arc-Large-scale continental subduction, oroclinal bending and back-arc spreading, Earth Planet. Sci. Lett., 75, 167-183.

Lacassin, R., et al. (2004), Large-scale geometry, offset and kinematic evolution of the Karakorum fault Tibet, Earth Planet. Sci. Lett., 219, 255-269.

Langille, J. M., M. J. Jessup, J. M. Cottle, D. Newell, and G. Seward (2010), Kinematic evolution fo the Ama Drime detachment: Insights into orogenparallel extension and exhumation fo the Ama Drime Massif Tibet-Nepal, J. Struct. Geol., 32, 900-919, doi:10.1016/j.jsg.2010.04.005.

Langille, J. M., M. J. Jessup, J. M. Cottle, G. Lederer, and T. Ahmad (2012), Timing of metamorphism, melting and exhumation of the Leo Pargil dome, northwest India, J. Metamorph. Geol., 30, 769-791, doi:10.1111/j.1525-1314.2012.00998.x.

Larson, K., R. Burgmann, R. Bilham, and J. Freymuller (1999), Kinematics of the India-Eurasia collision Zone from GPS measurements, J. Geophys. Res., 104, 1077-1093.

Lee, J., C. Hager, S. R. Wallis, D. F. Stockli, M. J. Whitehouse, M. Aoya, and Y. Wang (2011), Middle to late Miocene extremely rapid exhumation and thermal reequilibration in the Kungo Co rift, southern Tibet, Tectonics, 30, TC2007, doi:10.1029/2010TC002745.

Li, D., and A. Yin (2008), Orogen-parallel, active left-slip faults in the Eastern Himalaya: Implications for the growth mechanism of the Himalayan Arc, Earth Planet. Sci. Lett., 274, 258-267.

Loveless, J. P., and B. J. Meade (2011), Partitioning of localized and diffuse deformation in the Tibetan Plateau from joint inversions of geologic and geodetic observations, Earth Planet. Sci. Lett., 303, 11-24.

McCaffrey, R., and J. Nabelek (1998), Role of oblique convergence in the active deformation of the Himalayas and southern Tibet plateau, Geology, 26, 691-694.

Miller, C., R. Schuster, U. Klötzli, W. Frank, and F. Purtscheller (1999), Post-Collisional Potassic and Ultrapotassic Magmatism in SW Tibet: Geochemical and Sr-Nd-Pb-O Isotopic Constraints for Mantle Source Characteristics and Petrogenesis, J. Petrol., 40, 1399-1424.

Molnar, P., and H. Lyon-Caen (1988), Some simple physical aspects of the support, structure, and evolution of mountain belts, Geol. Soc. Am. Spec. Pap., 218, 179-207

Murphy, M. A. (2007), Isotopic characteristics of the Gurla Mandhata metamorphic core complex: Implications for the architecture of the Himalayan orogen, Geology, 35, 983-986.

Murphy, M. A., and P. W. Burgess (2006), Geometry, kinematics, and landscape characteristics of an active transtension zone, Karakoram fault system, Southwest Tibet, J. Struct. Geol., 28, 263-283.

Murphy, M. A., and P. Copeland (2005), Transtensional deformation in the central Himalaya and its role in accommodating growth of the Himalayan orogen, Tectonics, 24, TC4012, doi:10.1029/2004TC001659.

Murphy, M. A., and A. Yin (2003), Structural evolution and sequence of thrusting in the Tethyan fold-thrust belt and Indus-Yalu suture zone, southwest Tibet, Geol. Soc. Am. Bull., 115, 21-34.

Murphy, M. A., A. Yin, P. Kapp, T. M. Harrison, D. Ling, and G. Jinghui (2000), Southward propagation of the Karakoram fault system, southwest Tibet: Timing and magnitude of slip, Geology, 28, 451-454.

Murphy, M. A., A. Yin, P. Kapp, T. M. Harrison, C. E. Manning, F. J. Ryerson, D. Lin, and G. Jinghui (2002), Structural evolution of the Gurla Mandhata detachment system, southwest Tibet: Implications for the eastward extent of the Karakoram fault system, Geol. Soc. Am. Bull., $114,428-447$

Murphy, M. A., J. E. Saylor, and L. Ding (2009), Late Miocene topographic inversion in southwest Tibet based on integrated paleoelevation reconstructions and structural history, Earth Planet. Sci. Lett., 282, 1-9.

Murphy, M. A., V. Sanchez, and M. H. Taylor (2010), Syncollisional extension along the Indian-Asian suture zone, south-central Tibet: Implications for crustal deformation of Tibet, Earth Planet. Sci. Lett., 290, 233-243.

Nabelek, P. I., and J. L. Nabelek (2011), A strain-heating model for the seismic low-velocity zone alont the Main Himalaya Thrust, J. Himalayan Earth Sci., 44, 62-63.

Nabelek, J., G. Hetenyi, J. Vergne, S. Sapkota, B. Kafle, M. Jiang, H. Su, J. Chen, and B. S. Huang (2009), Underplating in the Himalaya-Tibet Collision Zone Revealed by the Hi-CLIMB Experiment, Science, 325, 1371-1374.

Nabelek, P. I., A. G. Whittington, and A. M. Hofmeister (2010), Strain heating as a mechanism for partial melting and ultraheigh temperature metamorphism in convergent orogens: Implications of temperature-dependent thermal diffusivity and rheology, J. Geophys. Res., 115, B12417, doi:10.1029/2010JB007727.

Peltzer, G., and P. Tapponnier (1988), Formation and evolution of strike-slip faults, rifts, and basins during the India-Asia Collision: An experimental approach, J. Geophys. Res., 93, 15,085, doi:10.1029/JB093iB12p15085.

Phillips, R. J., R. R. Parrish, and M. P. Searle (2004), Age constraints on ductile deformation and long-term slip rates along the Karakoram fault zone Ladakh, Earth Planet. Sci. Lett., 226, 305-319.

Pullen, A., P. Kapp, P. G. DeCelles, G. E. Gehrels, and L. Ding (2011), Cenozoic anatexis and exhumation of Tethyan Sequence rocks in the Xiao Gurla Range Southwest Tibet, Tectonophysics, 501, 28-40.

Ratschbacher, L., W. Frisch, G. Liu, and C. Chen (1994), Distributed deformation in southern and western Tibet during and after the India-Asia collision, J. Geophys. Res., 99, 19,917-19,945.

Reiners, P. W. (2005), Zircon (U-Th)/He Thermochronometry Thermochro-nology, Rev. Mineral. Geochem., 58, $151-176$.

Robinson, A. C. (2009), Geologic offsets across the northern Karakorum fault: Implications for its role and terrane correlations in the western Himalayan-Tibetan orogen, Earth Planet. Sci. Lett., 279, 123-130.

Robinson, A. C., A. Yin, and O. M. Lovera (2010), The role of footwall deformation and denudation in controlling cooling age patterns of detachment systems: An application to the Kongur Shan extensional system in the Eastern Pamir China, Tectonophysics, 496, 28-43.

Rutter, E. H., D. R. Faulkner, K. H. Brodie, R. J. Phillips, and M. P. Searle (2007), Rock deformation processes in the Karakoram fault zone Eastern Karakoram, Ladakh, NW India, J. Struct. Geol., 29, 1315-1326.

Sanchez, V., M. A. Murphy, W. R. Dupre, L. Ding, and R. Zhang (2010), Structural evolution of the Neogene Gar Basin, western Tibet: Implications for releasing bend development and drainage patters, Geol. Soc. Am. Bull., 122, 926-945.

Sanchez, V. I., M. A. Murphy, A. C. Robinson, T. J. Lapen, and M. T. Heizler (2013), Tectonic evolution of the Indian-Asia suture zone since Middle Eocene time, Lopukangri area, south-central Tibet, J. Asian Earth Sci., 62(30), 205-220.

Searle, M. P. (1996), Geological evidence against large-scale pre-Holocene offsets along the Karakoram Fault: Implications for the limited extrusion of the Tibetan plateau, Tectonics, 15, 171-186, doi:10.1029/95TC01693.

Searle, M. P., R. F. Weinberg, and W. J. Dunlap (1998), Transpressional tectonics along the Karakoram fault zone, northern Ladkh: Constraints on Tibetan extrusion Continental transpressional and transtensional tectonics, Geol. Soc. Spec. Publ., 135, 307-326.

Seeber, L., and J. G. Armbruster (1984), Some elements of continental subductions along the Himalayan front, Tectonophysics, 105, 263-278, doi:10.1016/0040-1951(84)90207-5. 
Seeber, L., and A. Pecher (1998), Strain partitioning along the Himalayan arc and the Nanga Parbat antiform, Geology, 26, 791-794. Stockli, D. F. (2005), Application of low-temperature thermochronometry to extensional tectonic settings, Rev. Mineral. Geochem., 58, 411- 448 .

Styron, R., M. Taylor, and K. Okoronkwo (2010), Database of active structures from the Indo-Asian Collision, Eos Trans. AGU, 91(20), 181-182, doi:10.1029/2010EO200001.

Styron, R. H., M. H. Taylor, and M. A. Murphy (2011), Oblique convergence, arc-parallel extension, and the role of strike-slip faulting in the High Himalaya, Geosphere, 7, 582-596.

Styron, R. H., M. H. Taylor, K. E. Sundell, D. F. Stockli, J. A. G. Oalmann, A. T. McCallister, D. Liu, A. Moller, and L. Ding (2013), Miocene initiation and acceleration of extension in the South Lunggar rift, western Tibet: Evolution of an active detachment system from structural mapping and (U-Th)/He thermochronology, Tectonics, 32, 880-907, doi:10.1002/tect.20053.

Tapponnier, P., G. Peltzer, A. Y. Le Dain, R. Armijo, and P. Cobbold (1982), Propagating extrusion tectonics in Asia: New insights from simple experiments with plasticine, Geology, 10, 611-616

Taylor, M., and A. Yin (2009), Active structures of the Himalayan-Tibetan orogen and their relationships to earthquake distribution, contemporary strain field, and Cenozoic volcanism, Geophere, 5, 199-214.

Taylor, M., A. Yin, F. J. Ryerson, P. Kapp, and L. Ding (2003), Conjugate strike-slip faulting along the Bangong-Nujiang suture zone accommodates coeval east-west extension and north-south shortening in the interior of the Tibetan Plateau, Tectonics, 22(4), 1044, doi:10.1029/2002TC001361.

Thiede, R., J. Arrowsmith, B. Bookhagen, M. McWilliams, E. Sobel, and M. Strecker (2006), Dome formation and extension in the Tethyan Himalaya Leo Pargil, northwest India, Geol. Soc. Am. Bull., 118, 635-650.

Valli, F., N. Arnaud, P. H. Leloup, E. R. Sobel, G. Maheo, R. Lacassin, S. Guillot, H. Li, P. Tapponnier, and Z. Xu (2007), Twenty million years a continuous deformation along the Karakorum fault, western Tibet: A thermochronological analysis, Tectonics, 26, TC4004, doi:10.1029/ 2005 TC001913.

Valli, F., et al. (2008), New U-Th/He constraints on timing of shearing and long-term slip rate on the Karakorum fault, Tectonics, 27, TC5007, doi:10.1029/2007TC002184.

Wang, S., and T. J. Wright (2012), Satellite geodetic imaging reveals internal deformation of western Tibet, Geophys. Res. Lett., 39, L07303, doi:10.1029/2012GL051222.

Wang, S., X. Fang, Q. Lai, D. Zheng, and Y. Wang (2009), New radiometric dating constrains the time for initiation of the Karakorum fault zone [KFZ] SW Tibet, Tectonophysics, 475, 503-513.

Wang, S., E. Wang, X. Fang, and Q. Lai (2011), U-Pb SHRIMP and 40Ar/39Ar ages constrain the deformation history of the Karakoram fault zone [KFZ] SW Tibet, Tectonophysics, 509, 208-217.

Wang, S., C. Wang, R. J. Phillips, M. A. Murphy, X. Fang, and Y. Yue (2012), Displacement along the Karakoram fault NW Himalaya, estimated from LA-ICP-MS U-PB dating of offset geologic markers, Earth Planet. Sci. Lett., 337-338, 156-163.

Wang, S., M. A. Murphy, R. J. Phillips, and C. Wang (2013), Reply to Comment on “Displacement along the Karakoram fault, NW Himalaya, estimated from LA-ICPMS U-Pb dating of offset geologic markers" published by Leloup et al. in EPSL, 2012", Earth Planet. Sci. Lett., 363, $246-248$.

Wolfe, M. R., and D. F. Stockli (2010), Zircon (U-Th)/He thermochronometry in the KTB drill hole Germany, and its implications for bulk He diffusion kinetics in zircon, Earth Planet. Sci. Lett., 295, 69-82.

Wright, T. J., B. Parsons, P. C. England, and E. J. Fielding (2004), InSAR observations of low slip rates on the major faults of western Tibet, Science, 305, 236-239.

Yin, A., P. A. Kapp, M. A. Murphy, C. E. Manning, T. M. Harrison, M. Grove, D. Lin, D. Xi-Guang, and W. Cun-Ming (1999), Significant late Negoene eastwest extension in northern Tibet, Geology, 27, 787-790.

Zhang, P. Z., Z. Shen, M. Wang, W. J. Gan, R. Burgmann, and P. Molnar (2004), Continuous deformation of the Tibetan Plateau from global positioning system data, Geology, 32, 809-812.

Zhang, R., M. A. Murphy, T. J. Lapen, V. Sanchez, and M. Heizler (2011), Late Eocene crustal thickening followed by Early-Lage Oligocene extension along the India-Asia suture zone: Evidence for cyclicity in the Himalayan orogen, Geosphere, 7, 1249-1268.

Zhu, B., W. S. F. Kidd, D. B. Rowley, B. S. Currie, and N. Shafique (2005), Age of Initiation of the India-Asia Collision in the East-Central Himalaya, J. Geol., 2005(113), 265-285. 